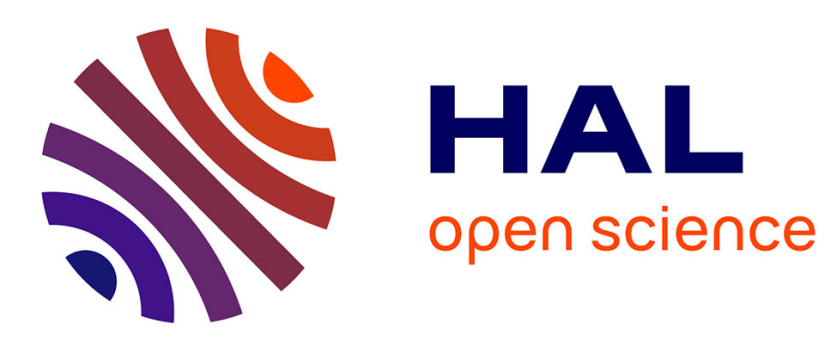

\title{
Atomic-resolution imaging of lattice imperfections in semiconductors by conjoined aberration-corrected HRTEM and exit-plane wavefunction retrieval
}

\author{
Karsten Tillmann, Lothar Houben, Andreas Thust
}

\section{- To cite this version:}

Karsten Tillmann, Lothar Houben, Andreas Thust. Atomic-resolution imaging of lattice imperfections in semiconductors by conjoined aberration-corrected HRTEM and exit-plane wavefunction retrieval. Philosophical Magazine, 2006, 86 (29-31), pp.4589-4606. 10.1080/14786430600675763 . hal00513689

\author{
HAL Id: hal-00513689 \\ https://hal.science/hal-00513689
}

Submitted on 1 Sep 2010

HAL is a multi-disciplinary open access archive for the deposit and dissemination of scientific research documents, whether they are published or not. The documents may come from teaching and research institutions in France or abroad, or from public or private research centers.
L'archive ouverte pluridisciplinaire HAL, est destinée au dépôt et à la diffusion de documents scientifiques de niveau recherche, publiés ou non, émanant des établissements d'enseignement et de recherche français ou étrangers, des laboratoires publics ou privés. 


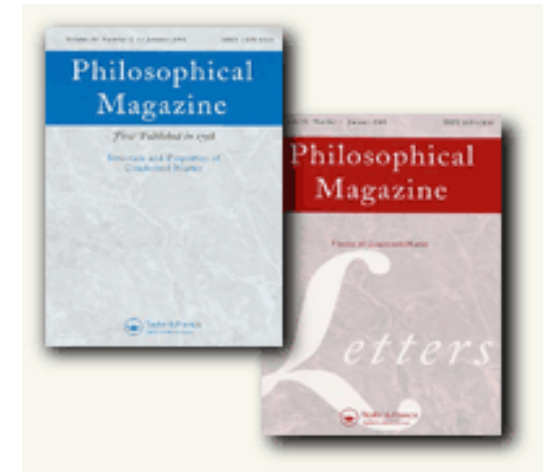

\section{Atomic-resolution imaging of lattice imperfections in semiconductors by conjoined aberration-corrected HRTEM and exit-plane wavefunction retrieval}

\begin{tabular}{|r|l|}
\hline Journal: & Philosophical Magazine \& Philosophical Magazine Letters \\
\hline Manuscript ID: & TPHM-05-Nov-0524.R3 \\
\hline Journal Selection: & Philosophical Magazine \\
\hline Author: & 05-Mar-2006 \\
\hline Complete List of Authors: & $\begin{array}{l}\text { Tillmann, Karsten; Forschungszentrum Juelich GmbH, Institut fuer } \\
\text { Festkoerperforschung } \\
\text { Houben, Lothar; Forschungszentrum Juelich GmbH, Institut fuer } \\
\text { Festkoerperforschung } \\
\text { Thust, Andreas; Forschungszentrum Juelich GmbH, Institut fuer } \\
\text { Festkoerperforschung }\end{array}$ \\
\hline Keywords: & $\begin{array}{l}\text { GaAs, GaN, HRTEM, dislocation structures, high-resolution imaging, } \\
\text { lattice defects, semiconductors, transmission electron microscopy }\end{array}$ \\
\hline Keywords (user supplied): & $\begin{array}{l}\text { spherical aberration correction, exit-plane wavefunction } \\
\text { reconstruction, dislocation core structure }\end{array}$ \\
\hline &
\end{tabular}

\section{ScholaroNE}


ATOMIC-RESOLUTION IMAGING OF LATTICE IMPERFECTIONS IN SEMICONDUCTORS BY CONJOINED ABERRATION-CORRECTED HRTEM AND EXIT-PLANE WAVEFUNCTION RETRIEVAL

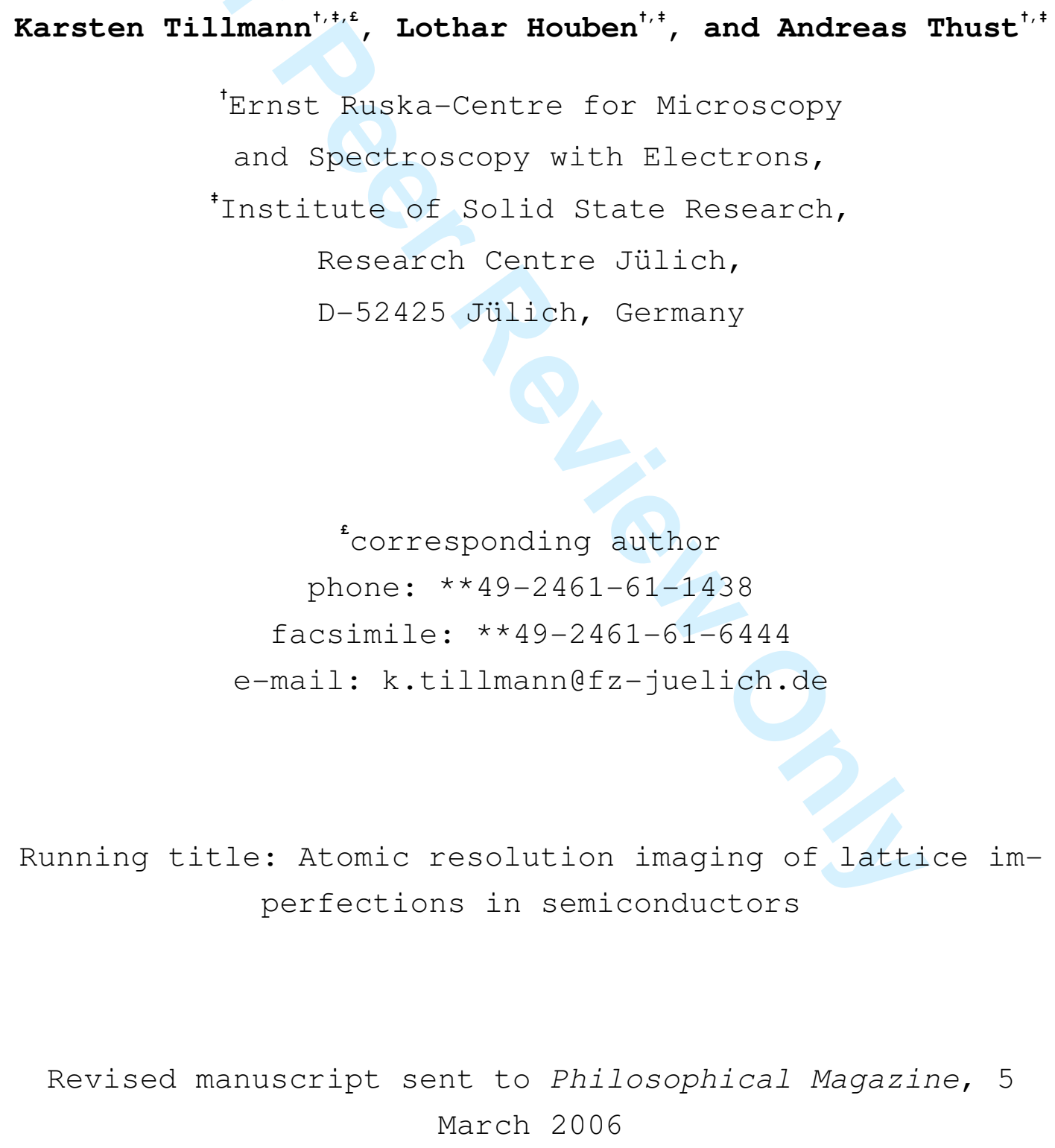


ABSTRACT

With the amelioration of the instrumental information limit and the simultaneous minimization of image delocalization, high-resolution transmission electron microscopy is presently enjoying exceeding popularity with respect to the atomic-scale imaging of lattice imperfections in solid state materials. In the present study the benefits derived from the deliberated combination of spherical aberration corrected imaging together with the numerical retrieval of the exit-plane wavefunction from a focal series of micrographs are illustrated by highlighting their combined use for the atomic-scale characterization of lattice defects frequently observed in common semiconductor materials. For these purposes, experimental analyses will review the core structure of Lomer dislocations at $\mathrm{In}_{0.3} \mathrm{Ga}_{0.7} \mathrm{As} / \mathrm{GaAs}-$ heterointerfaces and focus on atomic lattice displacements associated with extrinsic stacking fauls in GaAs, as well as on the core structure of chromium implantation induced Frank partial dislocations in GaN at directly interpretable contrast features. Supplementary, practical advantages of the retrieval of the exit-plane wavefunction for the subsequent numerical elimination of residual lens aberrations are demonstrated.

Key words: High-resolution transmission electron microscopy, spherical aberration correction, exit-plane wavefunction reconstruction, lattice defects, disloca- 


\section{GaAs, GaN}




\section{INTRODUCTION}

Over the past fifty years, transmission electron microscopy has become the technique of choice whenever structural properties associated with lattice imperfections in solid state materials have been investigated experimentally. Even though enormous progress has been made in the characterization of dislocations, stacking faults and internal boundaries in these common semiconductor materials, there is still much to learn about the precise atomic configuration in the vicinity of these structural imperfections. Above and beyond purely scientific curiosity this task is also of technological interest as further improvements in applied semiconductor research stand in need of an amended understanding of the interaction between structural and electronic properties with advancing miniaturization [i, ii]. To put it another way, the detailed evaluation of electronic features induced by lattice imperfections and the elucidation of the atomic structure of these imperfections are interdependent [iii, iv].

Recently of particular interest with high-resolution transmission electron microscopy is (i) the pursuit of sub-Ångström resolution at medium acceleration voltages [v, vi, vii] in close communion with (ii) the straightforward interpretability of micrographs [viii, ix, x] characterized by (iii) a rather low image delocalization in the vicinity of lattice imperfections [xi, xii]. Two sophisticated techniques to accomplish all three of these objectives simultaneously, not within reach during operation of "traditional" medium voltage instruments equipped with field emission gun emitters, have attracted much interest in recent years. 
On the one hand, double hexapole corrector elements enabling a compensation of detrimental lens aberrations are practically usable these days [xiii, xiv]. As a result, the aberration coefficient $C_{s}$ emerges as an additional parameter for extending the point resolution at phase contrast imaging conditions to the information limit together with the simultaneous maximization of phase contrast and the minimization of contrast delocalization [xv]. Moreover, when employing a negative $C_{s}$ value combined with a certain overfocus setting $z_{\text {opt }}>0$, not only a substantial contrast improvement is achieved but low-nuclear charge elements can also be imaged at bright atom contrast features in the vicinity of highnuclear charge atoms [viii, ix].

On the other hand, numerical techniques enable the restoration of the phase $\Phi(\boldsymbol{r})$ and the amplitude $A(\boldsymbol{r})$ of the exit-plane wavefunction from a focal series [xvi, xvii, xviii] or an illumination tilt direction series [xix, $x x]$ of experimental micrographs. By this means, all spatial information up to (focal series) or even beyond (tilt direction series) the axial information limit of the instrument can be retrieved, also allowing for a subsequent elimination of residual lens aberrations still present in aberration corrected microscopy [xxi, xxii, xxiii, xxiv].

The objective of the present contribution is to demonstrate the benefits accruing from the deliberated combination of both of these state-of-the-art techniques for the atomic scale imaging of structural imperfections in semiconductor materials. Special emphasize will be put on conceptual vantages for the characterization of lattice defects at atomic resolution and for 
the quantification of atomic column positions in the vicinity of lattice imperfections in semiconductor materials.

\section{MethodicAL BACKGROUND: NEgATIVE SPhericAL-ABERRATION Corrected Imaging in tandem with the Numerical Retrieval of THE EXIT-PLANE WAVEFUNCTION}

In aberration-corrected microscopy a high amount of negative phase contrast may be obtained simultaneously with a widely minimized image delocalization, when setting the constant of spherical aberration to an optimized value [ix, xv]

$$
C_{\mathrm{S}}=-64 / 27 \lambda^{-3} g_{\max }^{-4}
$$

and choosing an overfocus setting of the objective lens

$$
Z_{\mathrm{opt}}=16 / 9 \lambda^{-1} g_{\max }^{-2}
$$

with $\lambda$ and $1 / g_{\max }$ denoting the electron wavelength and information limit of the instrument, respectively. A corresponding set-up of the instrument yields direct interpretable high-resolution micrographs accompanied by a residual image delocalization of

$$
R=16 / 27 g_{\max }^{-1}
$$

Using an aberration corrected CM-200 FEG ST instrument, the aforementioned parameters amount to $Z_{\mathrm{opt}}=11.6 \mathrm{~nm}$, $C_{\mathrm{s}}=-40.6 \mathrm{~m}$ and $R=80 \mathrm{pm}$ when putting to use an electron wavelength of $\lambda=2.51 \mathrm{pm}$ and an information limit of $1 / g_{\max }=125 \mathrm{pm}$ with the latter value measured from a Young's fringe analysis of images taken with the microscope utilized in this study [XV]. 
The associated partially coherent phase contrast transfer function, displayed in Figure 1, extends up to $1 / g_{\max }$ and is characterized by a broad pass-band with a phase deviation close to $\cdot / 2$. Simplifying the imaging process to linear theory, a weak phase object is then imaged under bright atom contrast conditions [xxv] and a substantial contrast improvement is achieved since the linear phase contrast and the nonlinear dark field signal add rather than subtract as is usual when applying "traditional" high-resolution imaging modes [xxvi]. In the strict sense Eqns. (1) to (3) base upon a first order expansion of the aberration function, thus neglecting any detrimental impact of higher order lens aberrations. As the hexapole corrector of the CM-200 FEG ST allows for a sufficient correction of aberrations up to the third order [xxvii] and higher order aberrations will become relevant not until $1 / g_{\max }$ becomes smaller than 100 pm [xxviii, xxix], these aberrations do not need to be considered for the instrument under consideration.

The numerical retrieval of the exit-plane wavefunction $\Psi(\boldsymbol{r})$ from a focal series of micrographs provides auxiliary improvements as it is basically free from nonlinear imaging artifacts and the impact of the low frequency gap in information transfer is considerably reduced by the combination of many images taken at different foci. As improvements of the instrumental resolution limit still gather pace, this aspect is of special importance when imaging common semiconductors at atomic resolution since the structural information is predominantly carried by the low-frequency $\{111\}$ reflections. Furthermore, by extracting information from 
about $N=20 \ldots 30$ images, the signal-to-noise ratio at high spatial frequencies can be substantially increased. Even the application of small $C_{s}$ values, which is a prerequisite to obtain phase contrast, induces a parasitic delocalization whereas $\Psi(\boldsymbol{r})$ is ideally free from any delocalization induced artifacts.

Moreover, the availability of $\Psi(\boldsymbol{r})$ allows for the numerical a posterior measurement of residual lens aberrations. This aspect is of special practical importance as experience shows that not all aberrations of the microscope are sufficiently constant over the period of operation or cannot be determined before the experiment with sufficient accuracy. In detail, the measurement of even aberrations, $i$. e. the defocus $Z$ and the twofold astigmatism $A_{1}$, may be carried out by processing the weak signal originating from amorphous overlayers [xxi] while odd aberrations, $i$. e. the axial coma $B_{2}$ and the threefold astigmatism $A_{2}$, may be determined by sampling a variety of discrete aberration azimuths and magnitudes up to twice their arguable limits according to [xxvii] followed by handpicking those aberrations at which the symmetry properties of the corresponding $\Phi(\boldsymbol{r})$ and $\mathrm{A}(\boldsymbol{r})$ images correlate best with the supposed lattice structure [xxii]. Irrespective of this procedure even more sophisticated approaches are available to tackle the problem of measurement of odd aberrations $[\mathrm{xxx}]$.

Finally, since $\Psi(\boldsymbol{r})$ is complex-valued, we may evaluate "local" diffraction patterns from specimen areas as small as desired. When evaluated during operation of the microscope, the judgement of the symmetry properties of these "local" diffraction patterns is a most 
convenient tool for the proper orientation of specimen areas under investigation. By this means, a proper zone axis alignment of the samples with an accuracy below 3 mrad is ensured while the vast majority of samples aligned by "traditional" procedures demonstrates offzone-axis orientations above $10 \mathrm{mrads}$ [xxxi].

To set aright the common fallacy that the aforementioned benefits coming along with the restoration of the exit-plane wavefunction do not necessitate the use of spherical aberration corrected instrumentation but rather suggest an increase of the instrumental resolution solely, e. g. by monochromatisation of the electron source, it is emphasized that the a posterior measurement of residual lens aberrations requires a sufficient pre-reduction of the very same aberrations. Moreover, image delocalization in a non-corrected instrument will generally not be less than [xi]

$$
R_{L}=C_{S} / 4 \lambda^{3} g_{\max }^{3}
$$

and only to be within reach at Lichte's defocus of least confusion

$$
Z_{L}=-3 / 4 C_{S} \lambda^{2} g_{\max }^{2}
$$

which, alas, comes along with the great disadvantage that the phase contrast transfer function exhibits a large number of rapid contrast oscillations at medium spatial frequencies, and the corresponding contrast reversals make the resulting image quite difficult to interpret. Moreover, image delocalization according to Eqn. (4) does not only have a detrimental effect on the spread out of structural information in the vicinity of lattice imperfections but also to data acquisition at 
marginal areas of the recording medium. In other words, the rather large image delocalization of uncorrected instruments represents a serious limitation for the usable field of view of the charge-coupled device (CCD) camera detector.

\section{EXPERIMENTAL SETUP}

This study utilizes a Philips CM-200 FEG instrument equipped with a computer-controlled electromagnetic hexapole system for the correction of the spherical aberration of the objective lens [xxvii]. An optical coarse adjustment of the hexapole corrector elements was performed by analysing Zemlin tableaus taken from amorphous specimen areas with an incident electron beam tilted up to 20 mrad from the optical axis [xxxii] thus yielding an initial estimate on the quality of higherorder aberration corrections. Setting down an optimized negative $C_{\mathrm{s}}=-40 \mu \mathrm{m}$ the magnitudes of residual aberration coefficients were limited to $A_{1} \leq 2.0 \mathrm{~nm}$ for the two-fold astigmatism, to $A_{2} \leq 170 \mathrm{~nm}$ for the three-fold astigmatism and to $B_{2} \leq 55 \mathrm{~nm}$ for the axial coma [xxvii].

Focal series of about $N=20 \ldots 30$ images were recorded using a $1 \mathrm{k} \times 1 \mathrm{k}$ CCD camera system at a sampling rate of about $20 \mathrm{pm}$ per picture element. This corresponds to an image discretization well below the $\mathrm{Ny}-$ quist frequency with respect to half of the instrumental information limit $1 /\left(2 g_{\max }\right)=65 \mathrm{pm}$ and represents an adequate sampling to tap the full potential of the instrument facing that the modulation transfer function of many CCD cameras is known to exhibit a rather poor 
transfer at higher spatial frequencies. The focal range of each series included the focus setting with $Z_{\mathrm{opt}}=$ $11.6 \mathrm{~nm}$ for optimized phase contrast. From these series the exit-plane wavefunction was retrieved for the frequency band between $1 \mathrm{~nm}^{-1}$ and $8 \mathrm{~nm}^{-1}$ applying a doughnut-shaped restoration filter.

\section{Atomic Resolution Imaging at a Decent Signal-to-Noise Ratio: Core Structure of Misfit Dislocations at IN $_{0.3} \mathrm{GA}_{0.7}$ As /GAAS HETEROINTERFACES}

As a first materials science example of use, we report on the core structure of Lomer type pure edge dislocations [xxxiii] at the heterointerfaces between latticemismatched sphalerite semiconductors. In view of the multiplicity of conceivable lattice positions of the pulled out half-plane's terminal point, numerous core models have been proposed for this type dislocations [xxxiv, xxxv] but owing to the hitherto insufficient instrumental resolution previous high-resolution analyses required elaborate support by numerical image simulations merely to draw indirect conclusions on the corresponding dislocation core structures [xxxvi, xxxvii, xxxviii].

In the present analysis we focus on an In Ga $_{0.7} \mathrm{As}$ epilayer with a thickness of $35 \mathrm{~nm}$ that was grown by molecular beam epitaxy on a GaAs(001) substrate and subsequently overgrown with a GaAs capping layer of $55 \mathrm{~nm}$ in thickness whereby growth conditions were chosen as specified in a previous analysis [xxxix]. 
Figure 2(a) displays an optimum focus micrograph $I(\boldsymbol{r})$ of a Lomer dislocation with a Burgers circuit yielding a projected closing vector $\mathbf{b}=a / 2$ [1ํㅣㅁ] in the proximity of the interface between the $\operatorname{In}_{0.3} \mathrm{Ga}_{0.7} \mathrm{As}$ epilayer and the GaAs(001) substrate viewed with a [110] zone axis orientation of the sample. Far-off the dislocation core, the image contrast is dominated by clearly separated pairs of contrast dots constituting atomic dumbbells at distances of $a / 4 \cdot 141 \mathrm{pm}$ with $a=565 \mathrm{pm}$ denoting the lattice parameter of the substrate material.

However, two conspicuous contrast artifacts become evident from close inspection of the $z_{\mathrm{opt}}$ micrograph of the GaAs matrix displayed in Figure 2(e). Firstly, nonstructure related spurious intensity peaks are observed in between the atomic column positions which are due to an expended transfer of low spatial frequencies at severe phase offsets, especially of the strongly excited \{111\} beam amplitudes [xxvi]. Secondly, a kidney-shaped distortion of the dumbbells is observed. As can be shown from quantitative measurements on the associated "local" diffraction pattern displayed in Figure 2(b), these distortions are not due to any potential misorientation of the sample but instead result from parasitic lens aberrations amounting to $A_{1}=2.2 \mathrm{~nm}\left(83^{\circ}\right)$, $B_{2}=110 \mathrm{~nm}\left(83^{\circ}\right)$, and $A_{2}=150 \mathrm{~nm}\left(43^{\circ}\right)$ with the values in parentheses indicating the respective azimuth angles inclined with the [001] direction [xxxi]. For that reason, the phase $\Phi_{\text {unc }}(\boldsymbol{r})$ of exit-plane wavefunction retrieved from a focal series of $N=30$ micrographs and displayed in Figure $2(e)$ still shows distortions of the contrast dumbbells as long as these residual aberrations are not considered during the initial step of the 
reconstruction process. By contrast, the phase image $\Phi(\boldsymbol{r})$ is free of any distortions after the above specified aberrations $A_{i}$ and $B_{i}$ have been eliminated [xxxi].

Above and beyond this, the phase image is also richer in contrast compared to the $Z_{\mathrm{opt}}$ micrograph since there are practically no limitations with respect to the temporal and spatial coherence, thus providing a superior signal-to-noise ratio at high frequencies up to the information limit. Additionally, the retrieved phase benefits from the averaging of information from a complete series of micrographs. When quantifying the signal-to-noise-ratios $\sigma_{\Phi}$ and $\sigma_{\mathrm{I}}$ of the $\Phi(\boldsymbol{r})$ and $\mathrm{I}(\boldsymbol{r})$ images, respectively, by applying analysis procedures outlined in [xl] an improvement through the restoration of the exit-plane wavefunction by a factor of $\left(\sigma_{\Phi} / \sigma_{\mathrm{I}}\right)_{\exp }=$ 4.1 is obtained. This value is in excellent agreement with the ideal factor of $\left(\sigma_{\Phi} / \sigma_{\mathrm{I}}\right)_{\mathrm{th}}=\cdot N / 2=\cdot 30 / 2=3.9$ to be expected for information retrieval from a focal series of $N=30$ micrographs.

When also determining the polarity of the sample by the evaluation of a set of relevant reciprocal-space amplitudes from the "local" diffraction pattern and the subsequent trimming of simulated data, individual bright contrast dots in the $\Phi(\boldsymbol{r})$ image may be directly associated with gallium and arsenic columns [xxxi]. Corresponding atomic species are indicated in Figure $2(e)$.

The essential benefit of the combined use of the numerical retrieval of the exit-plane wavefunction with aberration-corrected imaging, however, becomes evident when having a closer look at the atomic structure of 
the dislocation core, which is basically not resolved in the single optimum focus image $I(\boldsymbol{r})$ but only in the amplitude and phase images, respectively. Both, $A(\mathbf{r})$ and $\Phi(\boldsymbol{r})$, show the atomic arrangement down to the dislocation core and reveal a detached atomic column at the point of intersection of extrapolated line positions indicated by the horizontal and vertical arrows. Relevant contrast features show mirror symmetry with respect to the medial (110) lattice plane indicated by the upright arrows. This global symmetry behaviour ranges down to the dislocation core.

From the exit-plane wavefunction a core structure model according to Figure $3(\mathrm{a})$ can be deduced as contrast features are intuitionally interpretable. The dislocation core shows some similarity to the glide set type model of a Lomer dislocation originally proposed by Hornstra [xxxiv] which is displayed in Figure 3(b) and characterized by an eight-atomic inner ring. The present analysis, however, reveals a less compact core, which is build up of a ten-sided polygon also traversing the detached atomic column with a coordination different from the surrounding matrix. This structure also deviates from rather complex mirror symmetric configurations observed at GaAs/Si interfaces [xxxvi, xli] and in Ge/Si heterosystems [xxxviii]. As surface effects in the vicinity of the highly strained dislocation core are likely to occur in thin electron microscopy specimens, the retrieved core structure may certainly deviate from that of a dislocation core embedded in bulk material. This problem, however, exists in any highresolution analysis concerned with lattice defects raising rather strong lattice distortions. 
Referring to a detailed discussion of the matter [xxxi] we briefly summarize the causal connections impeding the resolvability of the dislocation core in the $Z_{\text {opt }}$ image but evidently not in the retrieved exit-plane wavefunction. Numerical image calculations give clear evidence that this non-resolvability in the single I(r) image will neither be due to (i) the residual image delocalization or (ii) a decreased sample thickness in the vicinity of the dislocation core. Moreover, a potential impact of (iii) a locally inhomogeneous strain relaxation of the thin specimen may be ruled out since, otherwise, the $\mathrm{A}(\boldsymbol{r})$ and $\Phi(\boldsymbol{r})$ images would similarly be affected. Instead, the practically improved resolution of the amplitude and phase images will be rather due to (iv) an increased resistibility against time-dependant electron beam induced degeneration of the sample. While numerical retrieval techniques purge information from intrinsically unstable amorphous overlayers by averaging, a single $z_{\text {opt }}$ micrograph simply represents merely a snap-shot of a sample under illumination with a certain probability of an adverse atomic overlayer assembly during the time of exposure [xxxi].

\section{GeOMetric Linearity at High-LEVel Precession: LAtTice Distortions Associated With Dissociated $60^{\circ}$ Dislocations IN GAAS (110)}

In sphalerite materials $60^{\circ}$ dislocations with a Burgers vectors of type $\mathbf{b}=a / 2<0 \overline{1} 1>$ may dissociate into two partials bounding either an intrinsic or an extrinsic stacking fault [xlii, xliii, xliv, xlv]. The dissociation reaction is: 

$a / 2[0 \overline{1} 1]->$
$a / 6$
$[1 \overline{2} 1]+a / 6$
$[\overline{1} \overline{1} 2]$

with the Burgers vectors of both partials inclining angles of $30^{\circ}$ and $90^{\circ}$ with the dislocation line along the [110] direction, respectively. While most analyses at the outset of quantitative high-resolution microscopy were primarily concerned with distinguishing glide and shuffle set dislocations, i. e. discriminating whether dislocations cores lie on the narrowly or widely spaced \{111\} planes, by interpreting characteristic contrast features in the vicinity of the dislocations cores [xlvi, xlvii, xlviii], it is only recently that efforts have been put forth to investigate the core structure of $30^{\circ}$ and $90^{\circ}$ partials, both theoretically applying ab initio calculations [xlix, l, li] and experimentally employing advanced electron microscopy techniques [lii, liii].

In the following we shall focus on a dissociated $60^{\circ}$ dislocation bounding a double stacking fault ribbon. Figure $4(a)$ displays an optimum micrograph $I(\boldsymbol{r})$ of the terminating zone of such an extended dislocation in GaAs viewed along the crystallographic [110] direction. The image has been taken from the same specimen investigated in the previous section focusing on the GaAs capping layer in this case. The associated phase $\Phi(\boldsymbol{r})$ and amplitude $A(\boldsymbol{r})$ images displayed in Figure 4(b) and (c) allow for an accurate identification of the atomic arrangement of the stacking fault ribbons and, especially, the core structure of the dislocation terminating the defect.

As the overall lattice defect is composed of two adjacent (111) lattice planes showing similar misorienta- 
tions of the atomic dumbbells, it appears inceptively reasonable to describe the entire defect as a sequence of two adjoining likewise congenerous faulted fault ribbons. Both ribbons need then to be characterized by a projected component of the terminating Burgers vector amounting to $\mathbf{b}_{\text {proj }} / 2$ with $\mathbf{b}_{\text {proj }}$ denoting the closing vector of the entire defect when considering a projection to the (110) plane. Assuming a configuration build-up by $30^{\circ}$ partials, characterized by $\mathbf{b}_{30^{\circ-A}}=a / 6$ [12 1$]$ as well

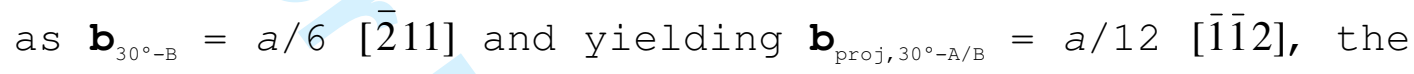
associated total lattice displacement along the [1 $1 \overline{1} 2]$ direction between the upper and the lower areas of the crystal far from the dislocation core should be $\Delta u_{\mathrm{ii} 2,30^{\circ}}=$ $2 \mathbf{b}_{\text {proj, } 30^{\circ}}=231 \mathrm{pm}$. This description is fully compatible with the definition of an extrinsic stacking fault bound by a $90^{\circ}$ partial with $\mathbf{b}_{90^{\circ}}=\mathbf{b}_{\text {proj,900}}=$ $\mathrm{a} / 6[\overline{1} \overline{1} 2]=231 \mathrm{pm}$ and $\mathrm{a} 30^{\circ}$ partial with $\mathbf{b}_{\text {proj,30 }}=$ a/12 [1]12] = $115 \mathrm{pm}$ at the opposite core position. Both of these limiting partials are in fact again superpositions of $30^{\circ}$ and $90^{\circ}$ partials separated by two atomic layers [45] with the extrinsicality expressing in two adjacent ribbons of $\{111\}$ lattice planes with differently aligned atomic [liv].

To check the validity of this model hypothesis, the elastic displacement component $u_{\overline{i 1} 2}$ along the [1112] direction has been evaluated from the $\Phi(\boldsymbol{r})$ image using geometrical phase analysis algorithms [lv]. Calculations have been performed applying Gaussian shaped masks with decay parameters $\sigma=\left|\mathbf{g}_{111}\right| / 3$ centred around the $\mathbf{g}_{111}$ beams thus averaging information over regions of about $r_{\text {dir }} \cdot 3$ / (2 $\left.\left|\mathbf{g}_{111}\right|\right) \cdot 490 \mathrm{pm}$ in radius at most [lvi]. The correspondingly measured $u_{i 12}(\boldsymbol{r})$ distribution is displayed in 
Figure 4(e) together with a line profile $u_{\overline{i 12}}\left(\boldsymbol{r}_{\overline{i i} 1}\right)$ ex-

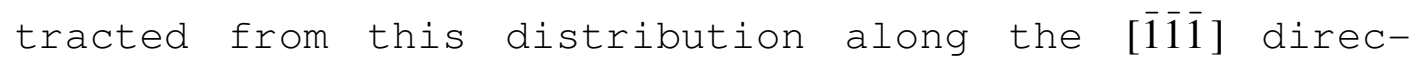
tion, cf. Figure 4(e). For the latter, data values have been averaged over a width of $1 \mathrm{~nm}$, $i$. e. over the width of the drawn-in dashed blue frame. The line profile shows two conspicuous plateaus separated by an abrupt discontinuity of $\cdot u_{i \overline{1} 2}=234 \mathrm{pm} \pm 60 \mathrm{pm}$. This quantity is in excellent agreement with the closing vector associated with the $90^{\circ}$ partial of an extrinsic stacking fault for which a total shift of lattice planes of $\Delta u_{\mathrm{ii}, 30^{\circ}}=2 \mathbf{b}_{\mathrm{proj}, 30^{\circ}-\mathrm{A} / \mathrm{B}}=231 \mathrm{pm}$ is expected, cf. above. We emphasize that the specified margin of error in the $\cdot u_{\mathrm{ii} 2}$ value rather reflects inhomogeneous lattice distortions of the sample but not the fundamental accuracy to quantify atomic column positions which may be measured to a precision of about a few picometres [lvii, lviii].

In Figure 4(d) single atomic columns, as allocated from the determination of the polarity of the sample, are superimposed to a magnified clipping of the phase image. The global displacement of the upper and lower crystal areas against each other is found to be mediated by the local re-organization of the atomic columns within those two (111) lattice planes forming the faulted double ribbon. Hence, the associated dislocation cores of both partials, indicated by red circles in the figure, also lie on these very same (111) planes. As these planes are narrowly spaced, the partial dislocations are of glide set type. Both dislocation cores are made up of gallium columns leading to a so-called $\beta$ dislocation [lix]. Moreover, the figure also reveals a symmetrical six-fold structure of the 
overall dislocation core, which is in perfect agreement with a $90^{\circ}$ partial dislocation composed of two $30^{\circ}$ partials separated by two atomic layers [xlv].

To put it a step further, we may also quantify local distortions of the atomic dumbbells across the faulted ribbon at some distance of the dislocation core. For these purposes contrast dot positions have been measured from the $5.5 \mathrm{~nm} \times 4.2 \mathrm{~nm}$ phase image $\Phi(\boldsymbol{r})$ area displayed in Figure 5(a), whose centre is $5.2 \mathrm{~nm}$ left of the dislocation core. For theses purposes a digital image processing algorithm [lx] has been applied which basically employs (i) dedicated image intensity thresholding operations, (ii) a centre-of-mass analysis inside individual bright contrast dots supported by (iii) adequate refining procedures as specified in [lxi, lxii]. From the measured contrast dot positions, the length and orientation of individual dumbbells have been calculated and normalized to mean values associated with the lower (planes no. 0 - 3) and upper (planes no. 11-13) areas of the $\Phi(\boldsymbol{r})$ image. For the improvement of statistics 15 data points have been averaged along the [1̄12] direction and median values are plotted in Figure 5(b) and (c) in dependence on specific (111) lattice plane positions.

As can be seen from both plots, the dumbbells on either side of the double ribbon rearrange roughly antisymmetric in the vicinity of the faulted (111) planes. In this context, the most conspicuous feature is that the dumbbelis of the bottommost lattice plane of the upper crystal domain (plane no. 8) are compressed to a length of $\langle d\rangle=133 \mathrm{pm} \pm 4 \mathrm{pm}$ and turn towards the double ribbon with a misorientation angle $\langle\delta\rangle \cdot 2.6^{\circ} \pm 1.3^{\circ} \mathrm{com}-$ 
pared to the reference lattice planes. In contrast, the uppermost dumbbells of the lower crystal area (plane no. 5) are stretched to a length of $\langle d\rangle=150 \mathrm{pm} \pm 4 \mathrm{pm}$ and bend away from the double ribbon at a misorientation angle of $\langle\delta\rangle \cdot-4.9^{\circ} \pm 1.5^{\circ}$.

In order to gauge whether the observed antisymmetric distortions represent a genuine structural property or not, potential implications of scattering and imaging artefacts need to be ruled out. Since a hypothetic global misalignment of the sample cannot give rise to any local torsion and dilatation of projected atomic dumbbells we may disenfranchise from this explanation. An argument of the same kind holds true of the potential impact of not fully compensated aberrations as they would take effect on the entire image and, hence, would distort all atomic dumbells of the same orientation equally. Strictly speaking any potential impact of both, on-axial and off-axial aberrations would need be evaluated separately. In practise this conceptional differentiation can, however, be neglected because of the rather limited field of view of the CCD detector and the semiamplanatic imaging properties of the double hexapole corrector [xiii]. Beyond electron-optical reasoning, a supposed strictly antisymmetric lattice distortion caused by a dedicated combination of higher order aberrations with the faulted ribbon incidentally acting as the symmetry plane, may be ruled out from a probability point of view because of an almost immense number of possible combinations which will not give raise to the observed distortion behaviour.

The observed alteration of atomic dumbbell lengths in the vicinity of stacking faults ribbons of about $10 \mathrm{pm}$ 
is in fair agreement with recent measurements of lattice distortions along intrinsic stacking fauls ribbons in heavily beryllium doped GaAs. This analysis revealed an average expansion of dumbbell lengths up to $158 \mathrm{pm}$ in the faulted lattice plane which was explained by the segregation of beryllium dopant atoms and the subsequent formation of antisite defects inside of the plane of the faulted ribbon [liii]. Additionally, lattice displacements around the central stacking fault of Z-shaped dipoles connecting two stair rod dislocations in indium doped GaAs revealed the very same antisymmetric distortion of dumbbell related contrast dots as were observed in the present analysis. Lateral displacements of gallium and arsenic atom pair related contrast features along the [1112] direction were found to be as much as $20 \%$ to $50 \%$ of the dumbbell length [lxiii].

As the specimen investigated in the present study was undoped we may, in the first instance, rule out the aforementioned explanations but only speculate about an indium diffusion from the underlying $\mathrm{In}_{0.3} \mathrm{Ga}_{0.7} \mathrm{As}$ layer to the GaAs layer along the faulted ribbon at most. As long as an inhomogeneous incorporation of indium atoms is not considered this approach, thus, cannot explain the observed lattice distortions. Admittedly, the antisymmetric distortions become perspicuous when considering the elastic distortions associated with the $90^{\circ}$ partial dislocations which basically follow the measured characteristics of the dumbbell's measured expansion and torsion but, alas, yield only alterations of dumbbells lengths smaller than \pm 3 pm and misorientation angles smaller than $\pm 0.4^{\circ}$ for the image area un- 
der investigation [liv]. Hence, a more likely explanation could be that the terminating partial dislocation biases the rearrangement of atomic columns in the vicinity of the faulted ribbons and that next but one neighbour interactions between different atomic species will indeed play an important role during this process.

\section{AtOMIC Structure OF Chromium Implantation Induced LAT- TICE DEFECTS IN GAN (1120)}

As a finale example, we report on dislocations introduced by chromium implantation into GaN. Implantation of transition metals into semiconductors is a candidate process for the fabrication of diluted magnetic semiconductors for spintronic applications [lxiv]. A byproduct of the implantation is the creation of ioninduced lattice defects: Extrinsic and intrinsic basal plane stacking faults, bounded by Frank partial or Shockley-Frank partial dislocations, are formed as the result of the precipitation of excess interstitials [liv, lxv].

The GaN films presented in this study were grown by metal organic chemical vapour deposition on (0001) oriented sapphire substrates under Si doping to a concentration of $2 \times 10^{17} \mathrm{~cm}^{-3}$. Cr+ ions were implanted with an energy of $200 \mathrm{kV}$ and a dose of $5 \times 10^{16} \mathrm{~cm}^{-2}$ at a temperature of $350{ }^{\circ} \mathrm{C}$ to avoid amorphization in the asimplanted samples. A high density of basal plane stacking faults is observed besides spherical Cr-rich precipitates in surface near regions after chromium implantation and rapid thermal annealing at $700{ }^{\circ} \mathrm{C}$ for 5 min in $\mathrm{N}_{2}$ atmosphere [lxvi]. 
The majority of the basal plane stacking faults is extrinsic bound by Frank partial dislocations with Burgers vector $\mathbf{b}=1 / 2$ [0001]. Figure 6 displays the amplitude image $A(\boldsymbol{r})$ and phase image $\Phi(\boldsymbol{r})$ of a perfect nanometre sized interstitial loop in the end of range depth of implantation. Gallium and nitrogen columns at a dumbbell distance of 114 pm, considerably smaller than the information limit of the instrument, are not fully resolved. Nonetheless the $\mathrm{N}$-polarity is unambiguously visible from the directly interpretable bright contrast in the phase image. The tetrahedral coordination across the faulted layer stacking is confirmed, asserting that no foreign chromium-gallium alloy phase is connected with the planar defects.

A magnified view of the core of a Frank partial dislocation at the terminating zone of a several $10 \mathrm{~nm}$ large dislocation loop is shown in the $Z_{\text {opt }}$ image and the $\Phi(\boldsymbol{r})$ image retrieved from a corresponding focal series displayed in Figure 7(a) and (b). Although the $I(\boldsymbol{r})$ and $\Phi(\boldsymbol{r})$ appear very similar at a first glance, the improved signal-to-noise ratio in the phase image enhances the visibility of the nitrogen positions and the dumbbell orientation up to the core of the dislocation. The phase image $\Phi(\boldsymbol{r})$ fortifies the presence of further nitrogen atoms within the core surrounded by the cage of the five marked gallium columns. The faint phase shift in $\Phi(\boldsymbol{r})$, in which non-linear image components and image delocalization are eliminated, indicates a nitrogen filled core in favour of a chromium or gallium rich core.

Based on these observations a tentative $5 / 7$ ring configuration for the core is presented in Figure 7(c). 
The nitrogen atoms shared by the 5- and 7-membered rings are threefold coordinated with Ga and N. Due to the wrong bond and the presence of unpaired electrons, this structure is not expected to be the most stable configuration. Since $\Phi(\boldsymbol{r})$ is retrieved from a focal series taken over a period of a few ten seconds, the image conceivably reflects a transient state of the core that gathers further interstitials during observation. The $Z_{\text {opt }}$ image level may therefore contain complementary but not necessarily identical information when compared to $\Phi(\boldsymbol{r})$. Indeed for the present case the $Z_{\mathrm{opt}}$ image with its inferior noise level compared to $\Phi(\boldsymbol{r})$ also complies an alternative structure which contains two wrong Ga-Ga bonds connecting threefold coordinated Ga atoms at positions $1-5$ and $2-3$, respectively.

\section{CONCLUSIONS}

In summary, a combination of negative spherical aberration imaging and the restoration of the exit-plane wavefunction from a focal series of micrographs has been applied for the investigation of lattice imperfections in semiconductor materials at atomic resolution and, in particular, for the extraction of quantitative information on local lattice distortions.

It has been demonstrated that recent improvements in the resolution power of transmission electron microscopes enable the imaging of finest structure details at directly interpretable contrast features also coming along with a widely minimized image delocalization when micrographs are taken under optimized imaging conditions. 
Additionally, the numerical retrieval of the exit-plane wavefunction allows for the elimination of artificial contrast features still visible in micrographs taken under optimized focusing conditions. Beyond it's genuine purpose, the retrieval of the exit-plane wavefunction was demonstrated to be a most suitable tool for the measurement and numerical correction of residual lens aberrations as well as for proper orientation of specimens during operation of the electron microscope.

Above and beyond the individual vantages coming along with either technique their combined use allows for the accurate identification of lattice imperfections in semiconductor materials at hitherto unequalled imaging. Experimental analyses conspicuously benefit from putting to use aberration corrected input data, i.e. from an increase of the instrumental information limit together with a decrease of image delocalization and a pre-reduction of lens aberrations, as well as from the complete elimination of residual aberrations by the numerical retrieval of the exit-plane wavefunction, which is also free from non-linear imaging artefacts and yields images characterized by a decent signal-to-noise ratio.

By means of the deliberated combination of both techniques, Lomer dislocation cores at In $_{0.3} \mathrm{Ga}_{0.7} \mathrm{As} / \mathrm{GaAs}$ heterointerfaces have been imaged at atomic resolution and directly identified as being composed of a mirror symmetric ten-atomic ring, thus rendering extensive image simulations superfluous. The quantification of local lattice distortions in the vicinity of a double stacking fault ribbon in GaAs by means of the analysis of experimental phase images $\Phi(\boldsymbol{r})$ brought clear evidence 
of an antisymmetric torsion and expansion of atomic dumbbells with respect to the faulted ribbon. A similar quantitative analysis focusing on lattice imperfections in GaN at atomic resolution based on the synergetic combination of negative spherical aberration corrected imaging and the numerical retrieval of the exit-plane wavefunction is presently merely hampered by the instrumental resolution but will come along with forthcoming aberration corrected sub-Ångström resolution microscopes.

\section{ACKNOWLEDGEMENTS}

The authors are grateful to Arno Förster and Vitaly Guzenko for making available the samples investigated in this study as well as Doris Meertens for indefatigable specimen preparation work. 
FIGURE CAPTIONS

Figure 1: Partially coherent contrast transfer function (PC CTF) together with the coherent transfer function (CTF) and the damping envelope (DE) assuming parameters $\lambda=2.51 \mathrm{pm}, C_{\mathrm{s}}=-40.6 \mu \mathrm{m}, Z=11.6 \mathrm{~nm}$ as well as $0.2 \mathrm{mrad}$ for the semi-angle of beam convergence and 6.4 $\mathrm{nm}$ for the half-width of the Gaussian spread of defocus. Arrows indicate positions of crystalline reflections in GaAs.

Figure 2: Analysis of a Lomer type dislocation with $\mathbf{b}=$ a/2 [1]10] at an $\operatorname{In}_{0.3} \mathrm{Ga}_{0.7} \mathrm{As} / \mathrm{GaAs}$ heterointerface. a: Optimum focus micrograph $\mathbf{I}(\boldsymbol{r})$ taken along the [110] zone axis orientation of the sample. b: "Local" diffraction pattern evaluated from the exit-plane wavefunction of the image area. c: Restored phase image $\Phi(\boldsymbol{r})$ and $\mathbf{d}$ : amplitude image $\mathrm{A}(\boldsymbol{r})$. e: Magnified clipping of the GaAs related image area together with the associated phase images $\Phi_{\text {unc }}(\boldsymbol{r})$ and $\Phi(\boldsymbol{r})$ obtained when neglecting and considering measured residual lens aberrations, respectively.

Figure 3: Dislocation core models for symmetrical Lomer dislocations. a: Model retrieved from the exit-plane wavefunction displayed in Figure 2 together with $\mathbf{b}$ : the symmetrical glide set type core model according to Hornstra [xxxiv].

Figure 4: Analysis of an extrinsic stacking fault in GaAs bound by two adjacent $30^{\circ}$ partial dislocations with $\mathbf{b}_{\text {proj }}=[\overline{1} \overline{1} 2]$ in total. a: High-resolution micrograph I $(r)$ taken under $Z_{\text {opt }}$ conditions. b: Phase image $\Phi(\boldsymbol{r})$ and 
c: amplitude image $\mathrm{A}(\boldsymbol{r})$ calculated from the associated focal series of micrographs. d: Magnified clipping of $\Phi(\boldsymbol{r})$ with the positions of atomic columns superimposed and the dislocation core positions of $30^{\circ}$ partials indicated by red circles. e: Contour representation of the lattice displacements along the [1112] direction with reference to the dashed red framed area. f: Displacement profile measured perpendicular to the faulted ribbon at the area indicated by the dashed blue frame.

Figure 5: Locally inhomogeneous distortions of the atomic dumbbells in the vicinity of the faulted double ribbon. a: Clipping from the phase image displayed in Figure 4(b) with atomic column positions superimposed and dumbbell distortions indicated exemplarily in dependence on specific positions along the [111] direction. b: Average projected bond length $\langle d\rangle$ and $\mathbf{c}$ : misorientation angle $\langle\delta\rangle$ of the dumbbells along the (111) direction. The lattice planes belonging to the double stacking fault ribbon are indicated in lighter grey colour.

Figure 6: Interstitial loop in Cr-implanted GaN viewed along the [11 $\overline{2} 0]$ direction. a: Amplitude image $\mathbf{A}(\mathbf{r})$ and b: phase image $\Phi(\boldsymbol{r})$ calculated from a focal series of micrographs. Simulated images of the periodic Npolarity GaN structure assuming a sample thickness of $3.2 \mathrm{~nm}$ at a resolution of $7.5 \mathrm{~nm}^{-1}$ are superimposed in the lower left part of the images. The stacking sequence is $A B A B C A B A B$, where the underlined part highlights sphalerite stacking.

Figure 7: Frank partial dislocation with the Burgers vector $\mathbf{b}=1 / 2$ [0001] projected along the [1120] direc- 
tion. a: Experimental micrograph $I(r)$ taken under $Z_{\text {opt }}$ conditions and $\mathbf{b}$ : correspondingly retrieved phase image $\Phi(\boldsymbol{r})$. c: $5 / 7$ ring configuration for the core of the Frank partial dislocation. 


\section{REFERENCES}

[i] H. Alexander and H. Teichler in Handbook of Semiconductor Technology, Vol 1, edited by K. A. Jackson and W. Schröter (Wiley-VCH, New York (U.S.), 2000), pp. 291-376.

[ii] R. Jones, Mater. Sci. Eng. B71 24 (2000).

[iii] P. B. Hirsch, Mater. Sci. Technol., 1(9) 666 $(1985)$.

[iv] S. P. Beckmann and D. C. Chrzan, Physica B 340-342 1001 (2003).

[v] C. Kisielowski, C. J. D. Hetherington, Y. C. Wang, R. Kilaas, M. A. O'Keefe, and A. Thust, Ultramicroscopy 89243 (2001).

[vi] M. A. O'Keefe, E.C. Nelson, E.C. Wang, and A. Thust, Phil. Mag. B 711861 (2001).

[vii] B. Freitag, S. Kujawa, P. M. Mul, J. Ringnalda, and P. C. Tiemeijer, Ultramicroscopy 102 209 (2005).

[viii] C. L. Jia, M. Lentzen, and K. Urban, Science 299870 (2003).

[ix] C. L. Jia, M. Lentzen, and K. Urban, Microscopy and Microanalysis 10174 (2004).

[x] J. L. Hutchison, J. M. Titchmarsh, D. J. H. Cockayne, R. C. Doole, C. J. D. Hetherington, A. I. Kirkland, and H. Sawada, Ultramicroscopy 1037 (2005).

[xi] H. Lichte, Ultramicroscopy 3813 (1991).

[xii] W. Coene and A. J. E. M. Jansen, Scan. Microsc. Suppl. 6379 (1992). 
[xiii] H. Rose, Optik 8519 (1990).

[xiv] M. Haider, H. Rose, S. Uhlemann, E. Schwan, B. Kabius, and K. Urban, Nature 392768 (1998).

[xv] M. Lentzen, B. Jahnen, C. L. Jia, A. Thust, K. Tillmann, and K. Urban, Ultramicroscopy 92233 $(2002)$.

[xvi] W. M. J. Coene, G. Janssen, M. Op de Beeck, and D. van Dyck, Phys. Rev. Lett. 693743 (1992).

[xvii] W. M. J. Coene, A. Thust, M. Op de Beeck, and D. van Dyck, Ultramicroscopy 64109 (1996).

[xviii] A. Thust, W. M. J. Coene, M. Op de Beeck, and D. van Dyck, Ultramicroscopy 64211 (1996).

[xix] A. I. Kirkland, W. O. Saxton, K. L. Chau, K. Tsuno, and M. Kawasaki, Ultramicroscopy 57355 (1995).

[xx] A. I. Kirkland, W. O. Saxton, and G. Chand, J. Electron Microsc. 111 (1997).

[xxi] A. Thust, M. H. F. Overwijk, W. M. J. Coene, and M. Lentzen, Ultramicroscopy 64249 (1996).

[xxii] A. Thust, C. L. Jia, and K. Urban, in Proceedings ICEM-15, Vol. 1, edited by R. Cross (Microscopy Society of Southern Africa, Durban, 2002), pp. 167-168.

[xxiii] R. R. Meyer, A. I. Kirkland, and W. O. Saxton, Ultramicroscopy 9289 (2002).

[xxiv] R. R. Meyer, A. I. Kirkland, and W. $O$. Saxton, Ultramicroscopy 99115 (2004).

[xxv] D. B. Williams and C. B. Carter, Transmission Electron Microscopy (Plenum Press, New York and London, 1996).

[xxvi] M. Lentzen, Ultramicroscopy 99211 (2004). 
[xxvii] S. Uhlemann and M. Haider, Ultramicroscopy 72 109 (1998).

[xxviii] M. A. O'Keefe, C. J. D. Hetherington,

Y. C. Wang, E. C. Nelson, J. H. Turner,

C. Kisielowski, J. O. Malm, R. Mueller,

J. Ringnalda, M. Pan, and A. Thust, Ultramicroscopy 89215 (2001).

[xxix] L. Y. Chang, F. R. Chen, A. I. Kirkland, and J. J. Kai, J. Electr. Microsc. 52359 (2003).

[xxx] R. R. Meyer, A. I. Kirkland, and W. O. Saxton, Ultramicroscopy 99115 (2004).

[xxxi] K. Tillmann, A. Thust, and K. Urban, Microscopy and Microanalysis 10185 (2004).

[xxxii] F. Zemlin, K. Weiss, P. Schiske, W. Kunath, and K. H. Herrmann, Ultramicroscopy 349 (1978).

[xxxiii] W. M. Lomer, Phil. Mag. 421327 (1951).

[xxxiv] J. Hornstra, J. Phys. Chem. Solids 5129 (1958)

[xxxv] A. Bourret, J. Dessaux and R. Renault, Phil. Mag. A 451 (1982).

[xxxvi] A. Vilà, A. Cornet, J. R. Morante, P. Ruterna, M. Loubradou, R. Bonnet, Y. González, and L. González, Phil. Mag. A 7585 (1995).

[xxxvii] D. Wang, H. Chen, F. H. Li, K. Kawasaki, T. Oikawa, Ultramicroscopy 93139 (2002).

[xxxviii] J. N. Stirman, P. A. Crozier, D. J. Smith, F. Phillipp, G. Brill and S. Sivananthan, Appl. Phys. Lett. 842530 (2004).

[xxxix] K. Tillmann and A. Förster, Thin Solid Films $36893(2000)$.

[xl] L. Houben, A. Thust, and K. Urban, Ultramicroscopy $106200(2006)$. 
[xli] S. Lopatin, S. J. Pennycook, J. Narayan, and G. Duscher, Appl. Phys. Lett. 812728 (2002).

[xlii] F. C. Frank, Phil. Mag. 42809 (1951).

[xliii] I. L. F. Ray and D. J. H. Cockayne, Proc. Roy. Soc. A 325543 (1971).

[xliv] P. Gai and A. Howie, Phil. Mag. 30939 (1974).

[xlv] S. Amelinckx, in Dislocations in Solids, Vol. 2, edited by F.R.N. Nabarro (North-Holland, Amsterdam, 1979), pp. 67-460.

[xlvi] A. Olsen and J. C. H. Spence, Phil. Mag. A 43 945 (1981).

[xlvii] M. Tanaka and B. Jouffrey, Phil. Mag. A 50 $733(1984)$.

[xlviii] B. C. de Cooman and D.B. Carter, phys. stat. sol. (a) 112473 (1989).

[xlix] R. W. Nunes, J. Bennetto, and D. Vanderbilt, Phys. Rev. B 5812563 (1998).

[1] J. F. Justo, R. W. Nunes, and L. V. C. Assali, J. Phys.: Condens. Matter 1412749 (2002).

[1i] S. P. Beckman, X. Xu, P. Specht, E. R. Weber, C. Kisielowski, and D. C. Chrzan, J. Phys.: Condens. Matter 1412673 (2002).

[lii] H. R. Kolar, J. C. H. Spence, and H. Alexander, Phys. Rev. Lett. 774031 (1996).

[liii] X. Xu, S. P. Beckmann, P. Specht, E. R. Weber, D. C. Chrzan, R. P. Ernie, I. Arslan, N. Browning, A. Bleloch, and C. Kisielowski, Phys. Rev. Lett. 95145501 (2005).

[liv] J. P. Hirth and J. Lothe, Theory of Dislocations, (McGraw Hill, New York (U.S), 1968). 
[lv] M. J. Hÿtch, E. Snoeck, and R. Kilaas, Ultramicroscopy 74131 (1998).

[lvi] J. L. Rouvière and E. Sarigiannidou, Ultramicroscopy 1061 (2005).

[lvii] C. L. Jia and A. Thust, Phys. Rev. Lett. 82 5052 (1999).

[lviii] M. J. Hÿtch, J. L. Putax, and J. M. Penisson, Nature 423279 (2003).

[lix] A. M. Gomez and P. B. Hirsch, Phil. Mag. A 38 $733(1978)$.

[lx] K. Tillmann, M. Lentzen, and R. Rosenfeld, Ultramicroscopy 83111 (2000).

[lxi] R. Kilaas, S. Paciornik, A. J. Schwartz, and L. E. Tanner, Journal of Computer-Assisted Microscopy 6129 (1994).

[lxii] H. Seitz, K. Ahlborn, M. Seibt, and W. Schröter, J. Microsc. 190184 (1997).

[lxiii] S. H. Lim, D. Shindo, I. Yonenaga, P. D. Brown, and C. J. Humphreys, Phys. Rev. Lett. 81 5350 (1998).

[lxiv] H. Ohno, Science 281951 (1998).

[lxv] V. Kirchner, H. Heinke, U. Birkle, S. Einfeld, D. Selke, and P. L. Ryder, Phys. Rev. B 58 15749 (1998).

[lxvi] V. A. Guzenko, N. Thillosen, A. Dahmen, R. Calarco, Th. Schäpers, L. Houben, M. Luysberg, B. Schineller, M. Heuken, and A. Kaluza, J. Appl. Phys. $965663(2004)$. 


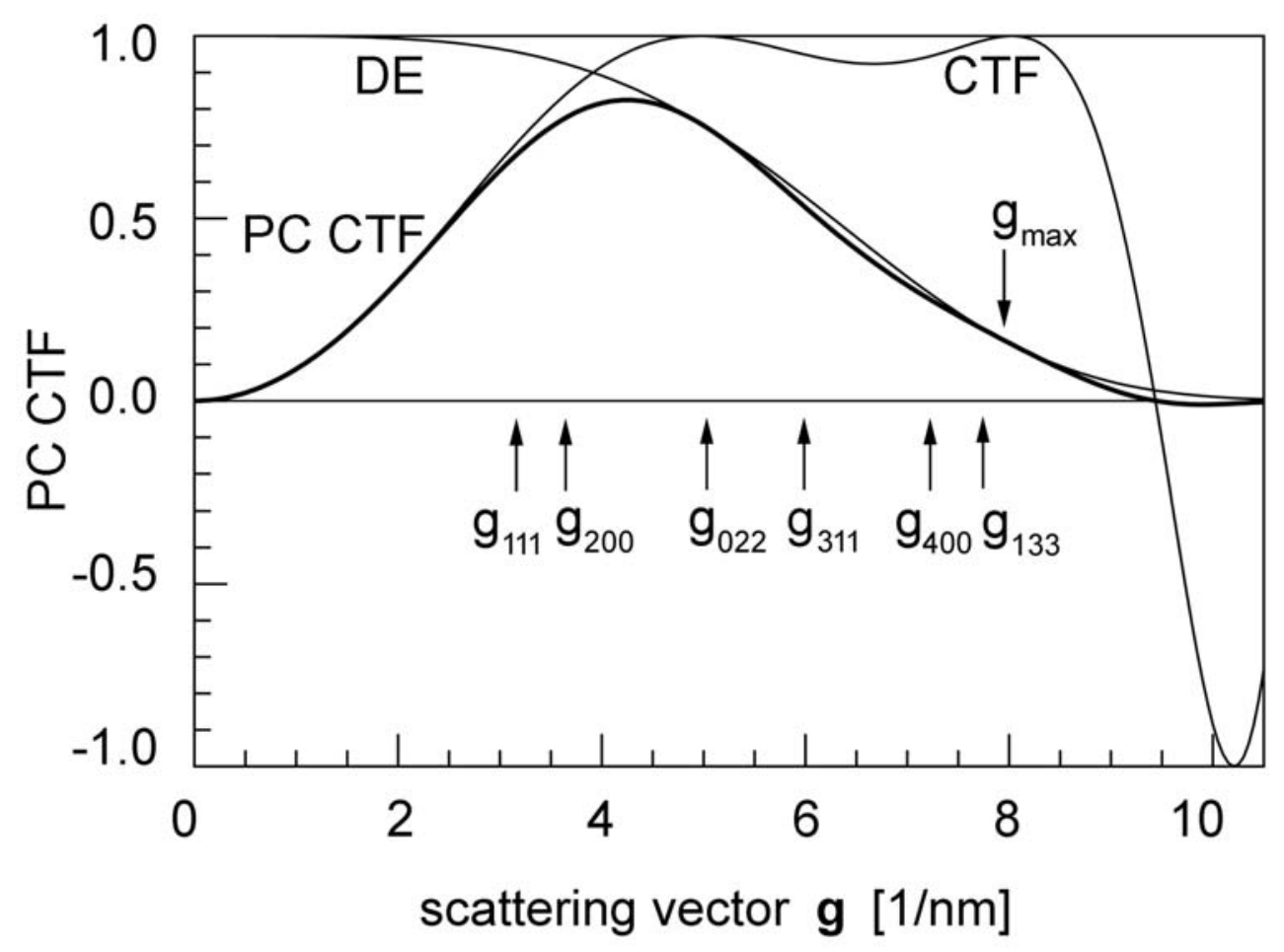

$160 \times 118 \mathrm{~mm}(300 \times 300 \mathrm{DPI})$ 


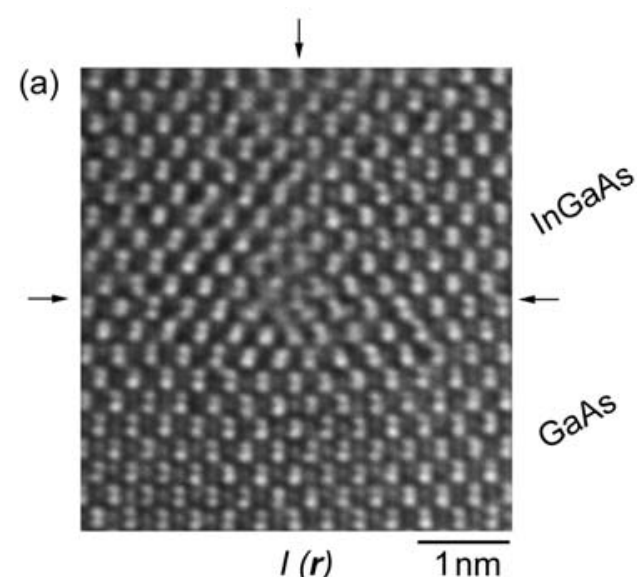

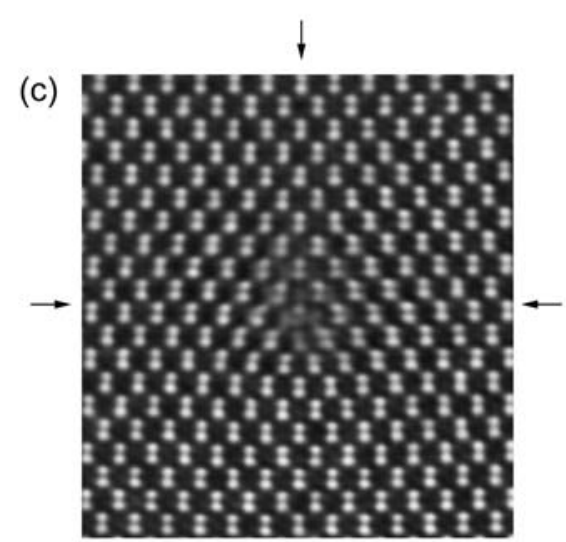

$\Phi(r)$

(e)

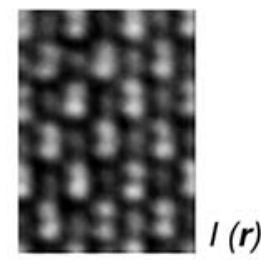

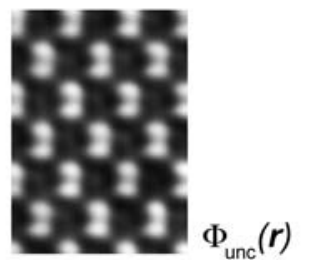

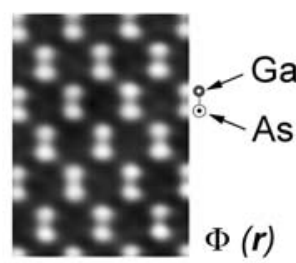

$160 \times 207 \mathrm{~mm}(600 \times 600 \mathrm{DPI})$

(b)

(d)
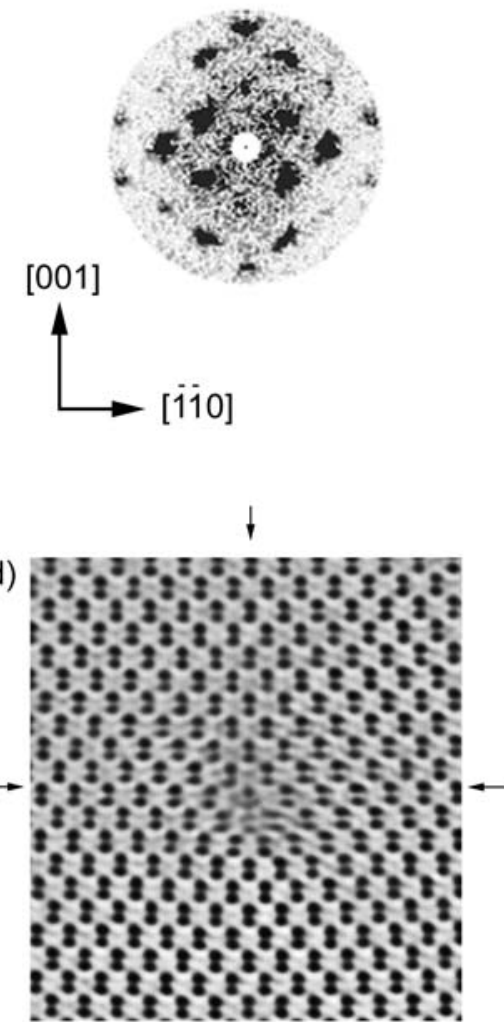

$A(r)$

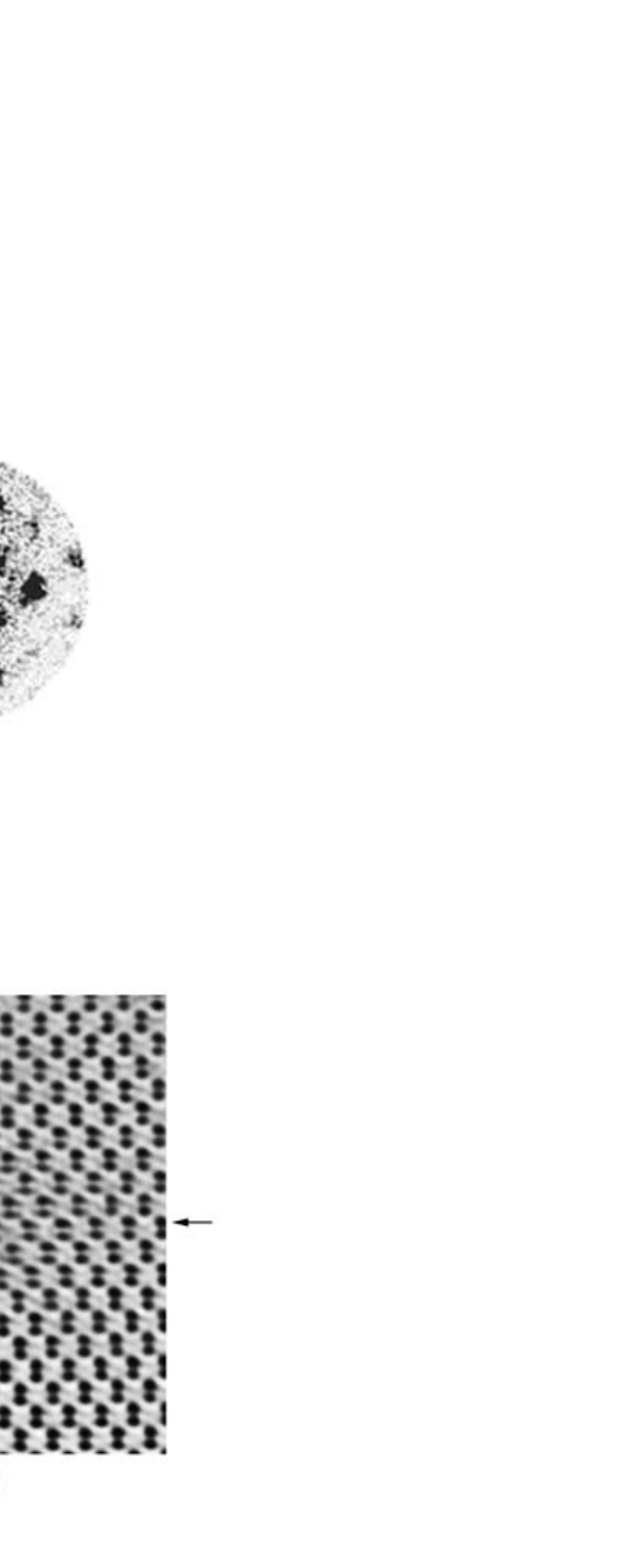



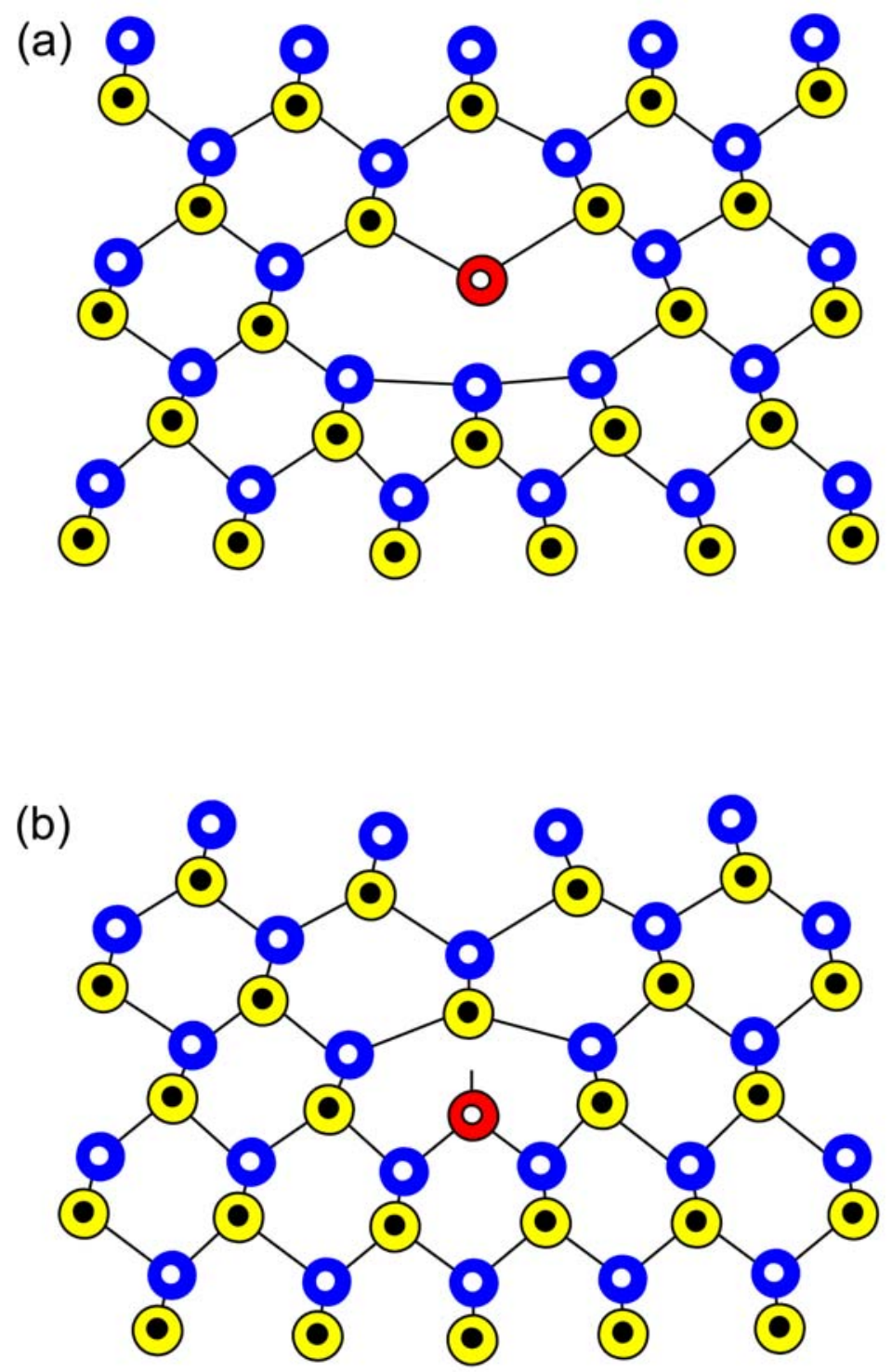

$139 \times 212 \mathrm{~mm}(600 \times 600 \mathrm{DPI})$ 


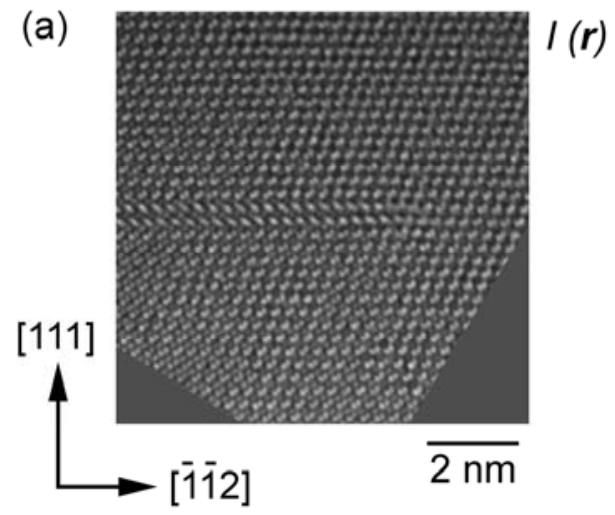

(b)

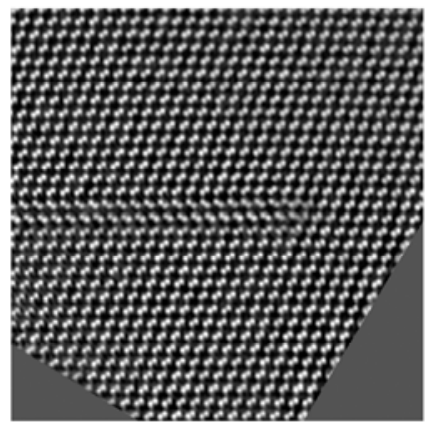

$\Phi(r)$

(e)

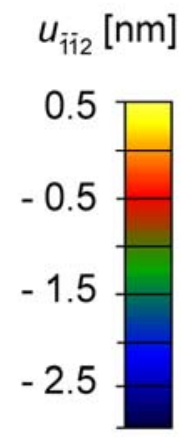

(d)

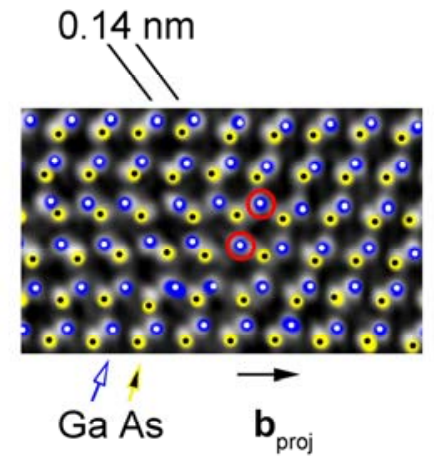

(c)

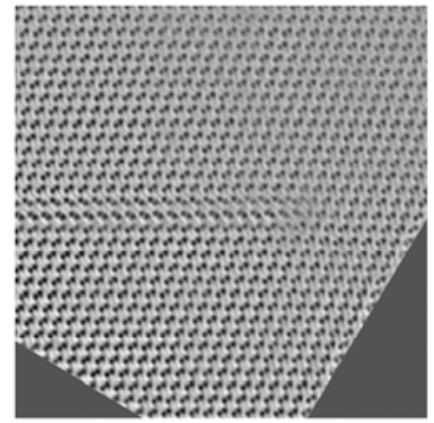

$A(r)$

(f)

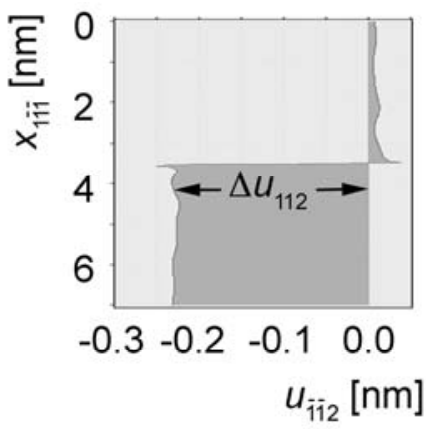

$160 \times 205 \mathrm{~mm}(600 \times 600 \mathrm{DPI})$ 

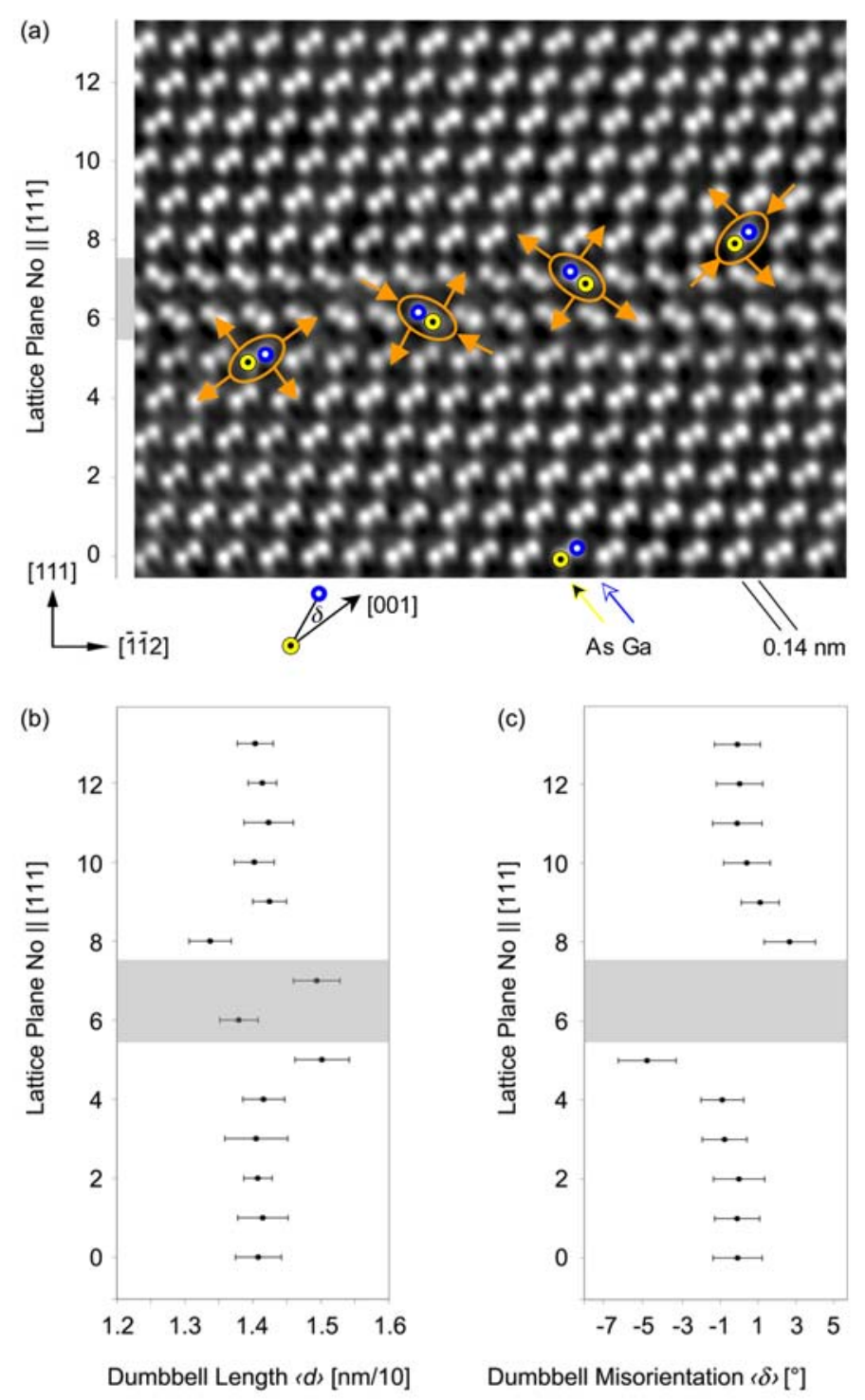

Dumbbell Misorientation $\langle\delta\rangle\left[^{\circ}\right]$ $139 \times 226 \mathrm{~mm}(600 \times 600 \mathrm{DPI})$ 
(a)

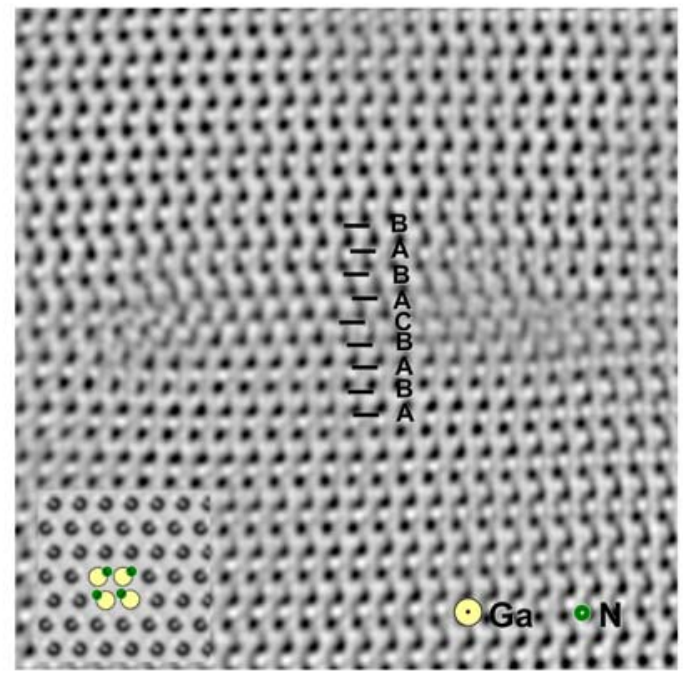

$A(r)$

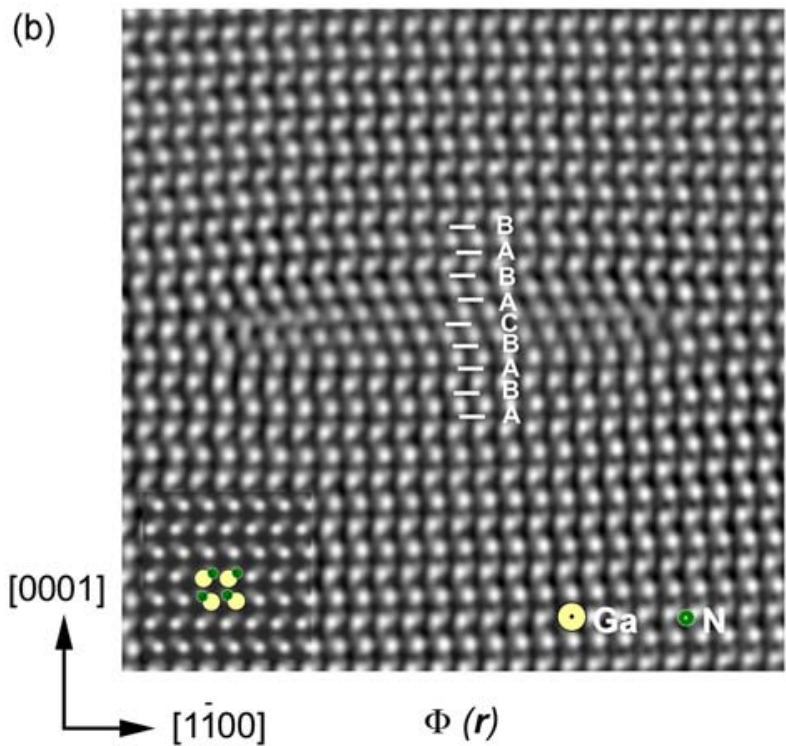

$120 \times 221 \mathrm{~mm}(600 \times 600 \mathrm{DPI})$

http://mc.manuscriptcentral.com/pm-pml 
(a)

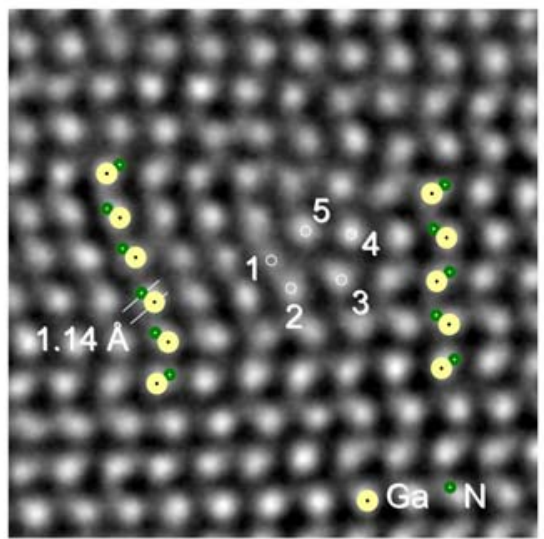

I (r) (b)

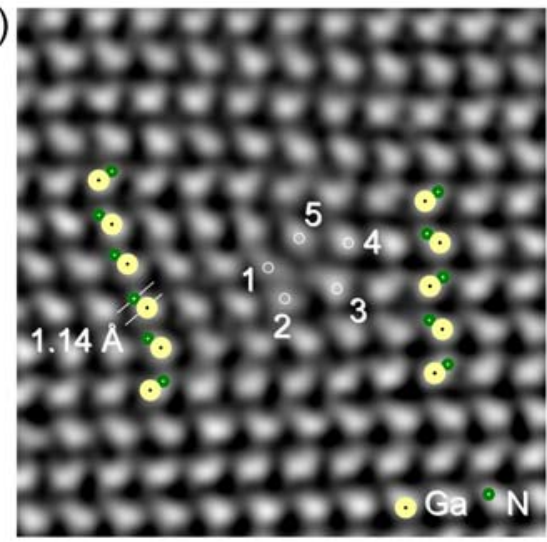

$\Phi(\boldsymbol{r})$

(c)

[1100]

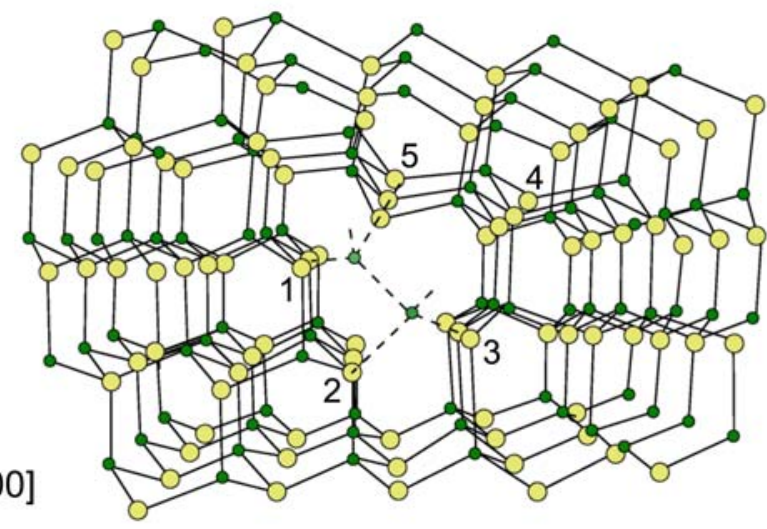

$\circ \mathrm{Ga}$

[0001]

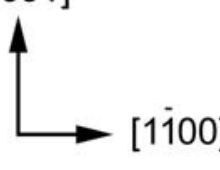

39

40

41

42

43

44

45

46

47

48

49

50

51

52

53

54

55

56

57

58

59

60

$139 \times 136 \mathrm{~mm}(600 \times 600 \mathrm{DPI})$ 


\title{
ATOMIC-RESOLUTION IMAGING OF LATTICE IM- PERFECTIONS IN SEMICONDUCTORS BY CON- JOINED ABERRATION-CORRECTED HRTEM AND EXIT-PLANE WAVEFUNCTION RETRIEVAL
}

\author{
Karsten Tillmann ${ }^{\dagger, \ddagger, £}$, Lothar Houben ${ }^{\dagger, \ddagger}$, and Andreas Thust ${ }^{\dagger, \neq}$ \\ ${ }^{\dagger}$ Ernst Ruska-Centre for Microscopy \\ and Spectroscopy with Electrons, \\ ${ }^{\ddagger}$ Institute of Solid State Research, \\ Research Centre Jülich, \\ D-52425 Jülich, Germany
}

\author{
${ }^{£}$ corresponding author \\ phone: ${ }^{* *} 49-2461-61-1438$ \\ facsimile: **49-2461-61-6444 \\ e-mail: k.tillmann@fz-juelich.de
}

Running title: Atomic resolution imaging of lattice imperfections in semiconductors

Revised manuscript sent to Philosophical Magazine, 5 March 2006 


\begin{abstract}
With the amelioration of the instrumental information limit and the simultaneous minimization of image delocalization, high-resolution transmission electron microscopy is presently enjoying exceeding popularity with respect to the atomic-scale imaging of lattice imperfections in solid state materials. In the present study the benefits derived from the deliberated combination of spherical aberration corrected imaging together with the numerical retrieval of the exit-plane wavefunction from a focal series of micrographs are illustrated by highlighting their combined use for the atomicscale characterization of lattice defects frequently observed in common semiconductor materials. For these purposes, experimental analyses will review the core structure of Lomer dislocations at $\operatorname{In}_{0.3} \mathrm{Ga}_{0.7}$ As/GaAs-heterointerfaces and focus on atomic lattice displacements associated with extrinsic stacking faults in GaAs, as well as on the core structure of chromium implantation induced Frank partial dislocations in GaN at directly interpretable contrast features. Supplementary, practical advantages of the retrieval of the exit-plane wavefunction for the subsequent numerical elimination of residual lens aberrations are demonstrated.
\end{abstract}

Key words: High-resolution transmission electron microscopy, spherical aberration correction, exit-plane wavefunction reconstruction, lattice defects, dislocation core structure, stacking faults, semiconductors, GaAs, GaN 


\section{INTRODUCTION}

Over the past fifty years, transmission electron microscopy has become the technique of choice whenever structural properties associated with lattice imperfections in solid state materials have been investigated experimentally. Even though enormous progress has been made in the characterization of dislocations, stacking faults and internal boundaries in these common semiconductor materials, there is still much to learn about the precise atomic configuration in the vicinity of these structural imperfections. Above and beyond purely scientific curiosity this task is also of technological interest as further improvements in applied semiconductor research stand in need of an amended understanding of the interaction between structural and electronic properties with advancing miniaturization $[1,2]$. To put it another way, the detailed evaluation of electronic features induced by lattice imperfections and the elucidation of the atomic structure of these imperfections are interdependent $[3,4]$.

Recently of particular interest with high-resolution transmission electron microscopy is (i) the pursuit of sub-Ångström resolution at medium acceleration voltages $[5,6,7]$ in close communion with (ii) the straightforward interpretability of micrographs [8, $9,10]$ characterized by (iii) a rather low image delocalization in the vicinity of lattice imperfections [11, 12]. Two sophisticated techniques to accomplish all three of these objectives simultaneously, not within reach during operation of "traditional" medium voltage instruments equipped with field emission gun emitters, have attracted much interest in recent years.

On the one hand, double hexapole corrector elements enabling a compensation of detrimental lens aberrations are practically usable these days [13, 14]. As a result, the aberration coefficient $\mathrm{C}_{S}$ emerges as an additional parameter for extending the point resolution at phase contrast imaging conditions to the information limit together with the simultaneous maximization of phase contrast and the minimization of contrast delocalization [15]. Moreover, when employing a negative $C_{S}$ value combined with a certain overfocus setting $Z_{\text {opt }}>0$, not only a substantial contrast improvement is achieved but low-nuclear charge elements can also be imaged at bright atom contrast features in the vicinity of high-nuclear charge atoms $[8,9]$. 
On the other hand, numerical techniques enable the restoration of the phase $\Phi(\mathbf{r})$ and the amplitude $\mathrm{A}(\mathbf{r})$ of the exit-plane wavefunction from a focal series $[16,17,18]$ or an illumination tilt direction series $[19,20]$ of experimental micrographs. By this means, all spatial information up to (focal series) or even beyond (tilt direction series) the axial information limit of the instrument can be retrieved, also allowing for a subsequent elimination of residual lens aberrations still present in aberration corrected microscopy $[21,22,23,24]$.

The objective of the present contribution is to demonstrate the benefits accruing from the deliberated combination of both of these state-of-the-art techniques for the atomic scale imaging of structural imperfections in semiconductor materials. Special emphasize will be put on conceptual vantages for the characterization of lattice defects at atomic resolution and for the quantification of atomic column positions in the vicinity of lattice imperfections in semiconductor materials.

\section{Methodical Background: Negative Spherical-Aberration Cor- RECTED IMAGING IN TANDEM WITH THE NUMERICAL RETRIEVAL OF THE EXIT-Plane Wavefunction}

In aberration-corrected microscopy a high amount of negative phase contrast may be obtained simultaneously with a widely minimized image delocalization, when setting the constant of spherical aberration to an optimized value $[9,15]$

$$
C_{S}=-64 / 27 \lambda^{-3} g_{\max }^{-4}
$$

and choosing an overfocus setting of the objective lens

$$
Z_{\text {opt }}=16 / 9 \lambda^{-1} g_{\max }^{-2}
$$

with $\lambda$ and $1 / g_{\max }$ denoting the electron wavelength and information limit of the instrument, respectively. A corresponding set-up of the instrument yields direct interpretable high-resolution micrographs accompanied by a residual image delocalization of

$$
R=16 / 27 g_{\max }^{-1} .
$$


Using an aberration corrected CM-200 FEG ST instrument, the aforementioned parameters amount to $Z_{\text {opt }}=11.6 \mathrm{~nm}, \mathrm{C}_{\mathrm{S}}=-40.6 \mu \mathrm{m}$ and $R=80 \mathrm{pm}$ when putting to use an electron wavelength of $\lambda=2.51 \mathrm{pm}$ and an information limit of $1 / \mathrm{g}_{\max }=$ $125 \mathrm{pm}$ with the latter value measured from a Young's fringe analysis of images taken with the microscope utilized in this study [15].

The associated partially coherent phase contrast transfer function, displayed in Figure 1 , extends up to $1 / g_{\max }$ and is characterized by a broad pass-band with a phase deviation close to $\pi / 2$. Simplifying the imaging process to linear theory, a weak phase object is then imaged under bright atom contrast conditions [25] and a substantial contrast improvement is achieved since the linear phase contrast and the nonlinear dark field signal add rather than subtract as is usual when applying "traditional" high-resolution imaging modes [26]. In the strict sense Eqns. (1) to (3) base upon a first order expansion of the aberration function, thus neglecting any detrimental impact of higher order lens aberrations. As the hexapole corrector of the CM-200 FEG ST allows for a sufficient correction of aberrations up to the third order [27] and higher order aberrations will become relevant not until $1 / g_{\max }$ becomes smaller than $100 \mathrm{pm}[28,29]$, these aberrations do not need to be considered for the instrument under consideration.

The numerical retrieval of the exit-plane wavefunction $\Psi(\mathbf{r})$ from a focal series of micrographs provides auxiliary improvements as it is basically free from nonlinear imaging artifacts and the impact of the low frequency gap in information transfer is considerably reduced by the combination of many images taken at different foci. As improvements of the instrumental resolution limit still gather pace, this aspect is of special importance when imaging common semiconductors at atomic resolution since the structural information is predominantly carried by the low-frequency $\{111\}$ reflections. Furthermore, by extracting information from about $N=20 \ldots 30$ images, the signal-to-noise ratio at high spatial frequencies can be substantially increased. Even the application of small $C_{S}$ values, which is a prerequisite to obtain phase contrast, induces a parasitic delocalization whereas $\Psi(\mathbf{r})$ is ideally free from any delocalization induced artifacts. 
Moreover, the availability of $\Psi(\mathbf{r})$ allows for the numerical a posterior measurement of residual lens aberrations. This aspect is of special practical importance as experience shows that not all aberrations of the microscope are sufficiently constant over the period of operation or cannot be determined before the experiment with sufficient accuracy. In detail, the measurement of even aberrations, i. e. the defocus $Z$ and the twofold astigmatism $A_{1}$, may be carried out by processing the weak signal originating from amorphous overlayers [21] while odd aberrations, i. e. the axial coma $B_{2}$ and the threefold astigmatism $A_{2}$, may be determined by sampling a variety of discrete aberration azimuths and magnitudes up to twice their arguable limits according to [27] followed by handpicking those aberrations at which the symmetry properties of the corresponding $\Phi(\mathbf{r})$ and $\mathrm{A}(\mathbf{r})$ images correlate best with the supposed lattice structure [22]. Irrespective of this procedure even more sophisticated approaches are available to tackle the problem of measurement of odd aberrations [30].

Finally, since $\Psi(\mathbf{r})$ is complex-valued, we may evaluate "local" diffraction patterns from specimen areas as small as desired. When evaluated during operation of the microscope, the judgement of the symmetry properties of these "local" diffraction patterns is a most convenient tool for the proper orientation of specimen areas under investigation. By this means, a proper zone axis alignment of the samples with an accuracy below $3 \mathrm{mrad}$ is ensured while the vast majority of samples aligned by "traditional" procedures demonstrates off-zone-axis orientations above 10 mrads [31].

To set aright the common fallacy that the aforementioned benefits coming along with the restoration of the exit-plane wavefunction do not necessitate the use of spherical aberration corrected instrumentation but rather suggest an increase of the instrumental resolution solely, e. g. by monochromatisation of the electron source, it is emphasized that the a posterior measurement of residual lens aberrations requires a sufficient pre-reduction of the very same aberrations. Moreover, image delocalization in a non-corrected instrument will generally not be less than [11]

$$
R_{L}=C_{S} / 4 \lambda^{3} g_{\max }^{3}
$$

and only to be within reach at Lichte's defocus of least confusion

$$
Z_{L}=-3 / 4 C_{S} \lambda^{2} g_{\max }^{2}
$$


which, alas, comes along with the great disadvantage that the phase contrast transfer function exhibits a large number of rapid contrast oscillations at medium spatial frequencies, and the corresponding contrast reversals make the resulting image quite difficult to interpret. Moreover, image delocalization according to Eqn. (4) does not only have a detrimental effect on the spread out of structural information in the vicinity of lattice imperfections but also to data acquisition at marginal areas of the recording medium. In other words, the rather large image delocalization of uncorrected instruments represents a serious limitation for the usable field of view of the charge-coupled device (CCD) camera detector.

\section{EXPERIMENTAL SETUP}

This study utilizes a Philips CM-200 FEG instrument equipped with a computercontrolled electromagnetic hexapole system for the correction of the spherical aberration of the objective lens [27]. An optical coarse adjustment of the hexapole corrector elements was performed by analysing Zemlin tableaus taken from amorphous specimen areas with an incident electron beam tilted up to $20 \mathrm{mrad}$ from the optical axis [32] thus yielding an initial estimate on the quality of higher-order aberration corrections. Setting down an optimized negative $C_{S}=-40 \mu \mathrm{m}$ the magnitudes of residual aberration coefficients were limited to $A_{1} \leq 2.0 \mathrm{~nm}$ for the two-fold astigmatism, to $A_{2} \leq 170 \mathrm{~nm}$ for the three-fold astigmatism and to $B_{2} \leq 55 \mathrm{~nm}$ for the axial coma [27].

Focal series of about $N=20 \ldots 30$ images were recorded using a $1 \mathrm{k}$ x $1 \mathrm{k}$ CCD camera system at a sampling rate of about $20 \mathrm{pm}$ per picture element. This corresponds to an image discretization well below the Nyquist frequency with respect to half of the instrumental information limit $1 /\left(2 g_{\max }\right)=65 \mathrm{pm}$ and represents an adequate sampling to tap the full potential of the instrument facing that the modulation transfer function of many CCD cameras is known to exhibit a rather poor transfer at higher spatial frequencies. The focal range of each series included the focus setting with $Z_{\text {opt }}=11.6 \mathrm{~nm}$ for optimized phase contrast. From these series the exit-plane wavefunction was retrieved for the frequency band between $1 \mathrm{~nm}^{-1}$ and $8 \mathrm{~nm}^{-1}$ applying a doughnut-shaped restoration filter. 


\section{Atomic Resolution Imaging at a Decent Signal-to-Noise Ratio: Core Structure of Misfit Dislocations at $\mathrm{IN}_{0.3} \mathrm{GA}_{0.7} \mathrm{As} / \mathrm{GAAs}$ Het- EROINTERFACES}

As a first materials science example of use, we report on the core structure of Lomer type pure edge dislocations [33] at the heterointerfaces between lattice-mismatched sphalerite semiconductors. In view of the multiplicity of conceivable lattice positions of the pulled out half-plane's terminal point, numerous core models have been proposed for this type dislocations $[34,35]$ but owing to the hitherto insufficient instrumental resolution previous high-resolution analyses required elaborate support by numerical image simulations merely to draw indirect conclusions on the corresponding dislocation core structures $[36,37,38]$.

In the present analysis we focus on an $\operatorname{In}_{0.3} \mathrm{Ga}_{0.7} \mathrm{As}$ epilayer with a thickness of $35 \mathrm{~nm}$ that was grown by molecular beam epitaxy on a GaAs(001) substrate and subsequently overgrown with a GaAs capping layer of $55 \mathrm{~nm}$ in thickness whereby growth conditions were chosen as specified in a previous analysis [39].

Figure 2(a) displays an optimum focus micrograph I(r) of a Lomer dislocation with a Burgers circuit yielding a projected closing vector $b=a / 2[\overline{1} \overline{1} 0]$ in the proximity of the interface between the $\operatorname{In}_{0.3} \mathrm{Ga}_{0.7} \mathrm{As}$ epilayer and the $\mathrm{GaAs}(001)$ substrate viewed with a [1ํㅣ zone axis orientation of the sample. Far-off the dislocation core, the image contrast is dominated by clearly separated pairs of contrast dots constituting atomic dumbbells at distances of $a / 4 \approx 141 \mathrm{pm}$ with $a=565 \mathrm{pm}$ denoting the lattice parameter of the substrate material.

However, two conspicuous contrast artifacts become evident from close inspection of the $Z_{\text {opt }}$ micrograph of the GaAs matrix displayed in Figure 2(e). Firstly, nonstructure related spurious intensity peaks are observed in between the atomic column positions which are due to an expended transfer of low spatial frequencies at severe phase offsets, especially of the strongly excited $\{111\}$ beam amplitudes [26]. Secondly, a kidney-shaped distortion of the dumbbells is observed. As can be shown from quantitative measurements on the associated "local" diffraction pattern dis- 
played in Figure 2(b), these distortions are not due to any potential misorientation of the sample but instead result from parasitic lens aberrations amounting to $A_{1}=2.2 \mathrm{~nm}\left(83^{\circ}\right), B_{2}=110 \mathrm{~nm}\left(83^{\circ}\right)$, and $A_{2}=150 \mathrm{~nm}\left(43^{\circ}\right)$ with the values in parentheses indicating the respective azimuth angles inclined with the [001] direction [31]. For that reason, the phase $\Phi_{\text {unc }}(\mathbf{r})$ of exit-plane wavefunction retrieved from a focal series of $N=30$ micrographs and displayed in Figure 2(e) still shows distortions of the contrast dumbbells as long as these residual aberrations are not considered during the initial step of the reconstruction process. By contrast, the phase image $\Phi(\mathbf{r})$ is free of any distortions after the above specified aberrations $A_{i}$ and $B_{i}$ have been eliminated [31].

Above and beyond this, the phase image is also richer in contrast compared to the $Z_{\text {opt }}$ micrograph since there are practically no limitations with respect to the temporal and spatial coherence, thus providing a superior signal-to-noise ratio at high frequencies up to the information limit. Additionally, the retrieved phase benefits from the averaging of information from a complete series of micrographs. When quantifying the signal-to-noise-ratios $\sigma_{\Phi}$ and $\sigma_{\mathrm{I}}$ of the $\Phi(\mathbf{r})$ and $\mathrm{I}(\mathbf{r})$ images, respectively, by applying analysis procedures outlined in [40] an improvement through the restoration of the exit-plane wavefunction by a factor of $\left(\sigma_{\Phi} / \sigma_{\mathrm{I}}\right)_{\exp }=4.1$ is obtained. This value is in excellent agreement with the ideal factor of $\left(\sigma_{\Phi} / \sigma_{\mathrm{I}}\right)_{\mathrm{th}}=\sqrt{\mathrm{N}} / 2=\sqrt{30} / 2=3.9$ to be expected for information retrieval from a focal series of $N=30$ micrographs.

When also determining the polarity of the sample by the evaluation of a set of relevant reciprocal-space amplitudes from the "local" diffraction pattern and the subsequent trimming of simulated data, individual bright contrast dots in the $\Phi(\mathbf{r})$ image may be directly associated with gallium and arsenic columns [31]. Corresponding atomic species are indicated in Figure 2(e).

The essential benefit of the combined use of the numerical retrieval of the exit-plane wavefunction with aberration-corrected imaging, however, becomes evident when having a closer look at the atomic structure of the dislocation core, which is basically not resolved in the single optimum focus image $\mathrm{I}(\mathbf{r})$ but only in the amplitude and phase images, respectively. Both, $\mathrm{A}(\mathbf{r})$ and $\Phi(\mathbf{r})$, show the atomic arrangement down to the dislocation core and reveal a detached atomic column at the point of intersec- 
tion of extrapolated line positions indicated by the horizontal and vertical arrows. Relevant contrast features show mirror symmetry with respect to the medial ( $\overline{1} \overline{1} 0)$ lattice plane indicated by the upright arrows. This global symmetry behaviour ranges down to the dislocation core.

From the exit-plane wavefunction a core structure model according to Figure 3(a) can be deduced as contrast features are intuitionally interpretable. The dislocation core shows some similarity to the glide set type model of a Lomer dislocation originally proposed by Hornstra [34] which is displayed in Figure 3(b) and characterized by an eight-atomic inner ring. The present analysis, however, reveals a less compact core, which is build up of a ten-sided polygon also traversing the detached atomic column with a coordination different from the surrounding matrix. This structure also deviates from rather complex mirror symmetric configurations observed at GaAs/Si interfaces [36, 41] and in Ge/Si heterosystems [38]. As surface effects in the vicinity of the highly strained dislocation core are likely to occur in thin electron microscopy specimens, the retrieved core structure may certainly deviate from that of a dislocation core embedded in bulk material. This problem, however, exists in any high-resolution analysis concerned with lattice defects raising rather strong lattice distortions.

Referring to a detailed discussion of the matter [31] we briefly summarize the causal connections impeding the resolvability of the dislocation core in the $Z_{\text {opt }}$ image but evidently not in the retrieved exit-plane wavefunction. Numerical image calculations give clear evidence that this non-resolvability in the single $I(\mathbf{r})$ image will neither be due to (i) the residual image delocalization or (ii) a decreased sample thickness in the vicinity of the dislocation core. Moreover, a potential impact of (iii) a locally inhomogeneous strain relaxation of the thin specimen may be ruled out since, otherwise, the $\mathrm{A}(\mathbf{r})$ and $\Phi(\mathbf{r})$ images would similarly be affected. Instead, the practically improved resolution of the amplitude and phase images will be rather due to (iv) an increased resistibility against time-dependant electron beam induced degeneration of the sample. While numerical retrieval techniques purge information from intrinsically unstable amorphous overlayers by averaging, a single $Z_{\text {opt }}$ micrograph simply represents merely a snap-shot of a sample under illumination with a certain probability of an adverse atomic overlayer assembly during the time of exposure [31]. 


\section{Geometric Linearity at High-Level Precession: Lattice Distor- TIONS ASSOCIATED WITH DisSOCIATED $60^{\circ}$ DislOCATIONS IN GAAs (110)}

In sphalerite materials $60^{\circ}$ dislocations with a Burgers vectors of type $b=a / 2<011>$ may dissociate into two partials bounding either an intrinsic or an extrinsic stacking fault $[42,43,44,45]$. The dissociation reaction is:

$$
a / 2[0 \overline{1} 1] \rightarrow a / 6[1 \overline{2} 1]+a / 6[\overline{1} \overline{1} 2]
$$

with the Burgers vectors of both partials inclining angles of $30^{\circ}$ and $90^{\circ}$ with the dislocation line along the [1ํㅣ direction, respectively. While most analyses at the outset of quantitative high-resolution microscopy were primarily concerned with distinguishing glide and shuffle set dislocations, i. e. discriminating whether dislocations cores lie on the narrowly or widely spaced $\{111\}$ planes, by interpreting characteristic contrast features in the vicinity of the dislocations cores [46, 47, 48], it is only recently that efforts have been put forth to investigate the core structure of $30^{\circ}$ and $90^{\circ}$ partials, both theoretically applying ab initio calculations $[49,50,51]$ and experimentally employing advanced electron microscopy techniques [52, 53].

In the following we shall focus on a dissociated $60^{\circ}$ dislocation bounding a double stacking fault ribbon. Figure 4(a) displays an optimum micrograph I(r) of the terminating zone of such an extended dislocation in GaAs viewed along the crystallographic [110] direction. The image has been taken from the same specimen investigated in the previous section focusing on the GaAs capping layer in this case. The associated phase $\Phi(\mathbf{r})$ and amplitude A(r) images displayed in Figure 4(b) and (c) allow for an accurate identification of the atomic arrangement of the stacking fault ribbons and, especially, the core structure of the dislocation terminating the defect.

As the overall lattice defect is composed of two adjacent (111) lattice planes showing similar misorientations of the atomic dumbbells, it appears inceptively reasonable to describe the entire defect as a sequence of two adjoining likewise congenerous faulted fault ribbons. Both ribbons need then to be characterized by a projected component of the terminating Burgers vector amounting to $b_{\text {proj }} / 2$ with $b_{\text {proj }}$ denoting the closing vector of the entire defect when considering a projection to the $(1 \overline{1} 0)$ 
plane. Assuming a configuration build-up by $30^{\circ}$ partials, characterized by $\mathrm{b}_{30^{\circ}-\mathrm{A}}=$ $a / 6$ [1 21$]$ as well as $b_{30^{\circ}-\mathrm{B}}=a / 6[\overline{2} 11]$ and yielding $b_{\text {proj, } 30^{\circ}-\mathrm{A} / \mathrm{B}}=a / 12$ [ $\left.\overline{1} \overline{1} 2\right]$, the associated total lattice displacement along the [1112] direction between the upper and the lower areas of the crystal far from the dislocation core should be $\Delta u_{\overline{\mathrm{i}} 2,30^{\circ}}=$ $2 b_{\text {proj,30 }}=231 \mathrm{pm}$. This description is fully compatible with the definition of an extrinsic stacking fault bound by a $90^{\circ}$ partial with $b_{90^{\circ}}=b_{\text {proj, } 90^{\circ}}=a / 6[\overline{1} \overline{1} 2]=231 \mathrm{pm}$ and a $30^{\circ}$ partial with $b_{\text {proj,30 }}=a / 12[\overline{1} \overline{1} 2]=115 \mathrm{pm}$ at the opposite core position. Both of these limiting partials are in fact again superpositions of $30^{\circ}$ and $90^{\circ}$ partials separated by two atomic layers [45] with the extrinsicality expressing in two adjacent ribbons of $\{111\}$ lattice planes with differently aligned atomic [54].

To check the validity of this model hypothesis, the elastic displacement component $u_{\overline{i 1} 2}$ along the [ $\left.\overline{1} 12\right]$ direction has been evaluated from the $\Phi(\mathbf{r})$ image using geometrical phase analysis algorithms [55]. Calculations have been performed applying Gaussian shaped masks with decay parameters $\sigma=\left|\mathbf{g}_{111}\right| / 3$ centred around the $\mathbf{g}_{111}$ beams thus averaging information over regions of about $r_{\mathrm{dir}} \approx 3 /\left(2\left|\mathbf{g}_{111}\right|\right) \approx 490 \mathrm{pm}$ in radius at most [56]. The correspondingly measured $u_{\mathrm{ii2}}(\mathbf{r})$ distribution is displayed in Figure 4(e) together with a line profile $u_{\overline{1 i 2}}\left(\mathbf{r}_{\overline{1} 1 \overline{1}}\right)$ extracted from this distribution along the $[\overline{1} \overline{1} \overline{1}]$ direction, cf. Figure $4(\mathrm{e})$. For the latter, data values have been averaged over a width of $1 \mathrm{~nm}$, i. e. over the width of the drawn-in dashed blue frame. The line profile shows two conspicuous plateaus separated by an abrupt discontinuity of $\Delta u_{\mathrm{i} 12}=234 \mathrm{pm} \pm 60 \mathrm{pm}$. This quantity is in excellent agreement with the closing vector associated with the $90^{\circ}$ partial of an extrinsic stacking fault for which a total shift of lattice planes of $\Delta u_{\bar{i} 2,30^{\circ}}=2 \mathrm{~b}_{\text {proj,30-A } / \mathrm{B}}=231 \mathrm{pm}$ is expected, cf. above. We emphasize that the specified margin of error in the $\Delta u_{\text {ii } 2}$ value rather reflects inhomogeneous lattice distortions of the sample but not the fundamental accuracy to quantify atomic column positions which may be measured to a precision of about a few picometres $[57,58]$.

In Figure 4(d) single atomic columns, as allocated from the determination of the polarity of the sample, are superimposed to a magnified clipping of the phase image. The global displacement of the upper and lower crystal areas against each other is found to be mediated by the local re-organization of the atomic columns within those two (111) lattice planes forming the faulted double ribbon. Hence, the associ- 
ated dislocation cores of both partials, indicated by red circles in the figure, also lie on these very same (111) planes. As these planes are narrowly spaced, the partial dislocations are of glide set type. Both dislocation cores are made up of gallium columns leading to a so-called $\beta$ dislocation [59]. Moreover, the figure also reveals a symmetrical six-fold structure of the overall dislocation core, which is in perfect agreement with a $90^{\circ}$ partial dislocation composed of two $30^{\circ}$ partials separated by two atomic layers [45].

To put it a step further, we may also quantify local distortions of the atomic dumbbells across the faulted ribbon at some distance of the dislocation core. For these purposes contrast dot positions have been measured from the $5.5 \mathrm{~nm} \times 4.2 \mathrm{~nm}$ phase image $\Phi(\mathbf{r})$ area displayed in Figure 5(a), whose centre is $5.2 \mathrm{~nm}$ left of the dislocation core. For theses purposes a digital image processing algorithm [60] has been applied which basically employs (i) dedicated image intensity thresholding operations, (ii) a centre-of-mass analysis inside individual bright contrast dots supported by (iii) adequate refining procedures as specified in [61, 62]. From the measured contrast dot positions, the length and orientation of individual dumbbells have been calculated and normalized to mean values associated with the lower (planes no. 0 - 3) and upper (planes no. 11-13) areas of the $\Phi(\mathbf{r})$ image. For the improvement of statistics 15 data points have been averaged along the [1̄12] direction and median values are plotted in Figure 5(b) and (c) in dependence on specific (111) lattice plane positions.

As can be seen from both plots, the dumbbells on either side of the double ribbon rearrange roughly antisymmetric in the vicinity of the faulted (111) planes. In this context, the most conspicuous feature is that the dumbbells of the bottommost lattice plane of the upper crystal domain (plane no. 8) are compressed to a length of $\langle d\rangle=133 \mathrm{pm} \pm 4 \mathrm{pm}$ and turn towards the double ribbon with a misorientation angle $\langle\delta\rangle \approx 2.6^{\circ} \pm 1.3^{\circ}$ compared to the reference lattice planes. In contrast, the uppermost dumbbells of the lower crystal area (plane no. 5) are stretched to a length of $\langle d\rangle=150 \mathrm{pm} \pm 4 \mathrm{pm}$ and bend away from the double ribbon at a misorientation angle of $\langle\delta\rangle \approx-4.9^{\circ} \pm 1.5^{\circ}$. 
In order to gauge whether the observed antisymmetric distortions represent a genuine structural property or not, potential implications of scattering and imaging artefacts need to be ruled out. Since a hypothetic global misalignment of the sample cannot give rise to any local torsion and dilatation of projected atomic dumbbells we may disenfranchise from this explanation. An argument of the same kind holds true of the potential impact of not fully compensated aberrations as they would take effect on the entire image and, hence, would distort all atomic dumbbells of the same orientation equally. Strictly speaking any potential impact of both, on-axial and offaxial aberrations would need be evaluated separately. In practise this conceptional differentiation can, however, be neglected because of the rather limited field of view of the CCD detector and the semiamplanatic imaging properties of the double hexapole corrector [13]. Beyond electron-optical reasoning, a supposed strictly antisymmetric lattice distortion caused by a dedicated combination of higher order aberrations with the faulted ribbon incidentally acting as the symmetry plane, may be ruled out from a probability point of view because of an almost immense number of possible combinations which will not give raise to the observed distortion behaviour.

The observed alteration of atomic dumbbell lengths in the vicinity of stacking faults ribbons of about $10 \mathrm{pm}$ is in fair agreement with recent measurements of lattice distortions along intrinsic stacking faults ribbons in heavily beryllium doped GaAs. This analysis revealed an average expansion of dumbbell lengths up to $158 \mathrm{pm}$ in the faulted lattice plane which was explained by the segregation of beryllium dopant atoms and the subsequent formation of antisite defects inside of the plane of the faulted ribbon [53]. Additionally, lattice displacements around the central stacking fault of Z-shaped dipoles connecting two stair rod dislocations in indium doped GaAs revealed the very same antisymmetric distortion of dumbbell related contrast dots as were observed in the present analysis. Lateral displacements of gallium and arsenic atom pair related contrast features along the [1112] direction were found to be as much as $20 \%$ to $50 \%$ of the dumbbell length [63].

As the specimen investigated in the present study was undoped we may, in the first instance, rule out the aforementioned explanations but only speculate about an indium diffusion from the underlying $\operatorname{In}_{0.3} \mathrm{Ga}_{0.7} \mathrm{As}$ layer to the GaAs layer along the faulted ribbon at most. As long as an inhomogeneous incorporation of indium at- 
oms is not considered this approach, thus, cannot explain the observed lattice distortions. Admittedly, the antisymmetric distortions become perspicuous when considering the elastic distortions associated with the $90^{\circ}$ partial dislocations which basically follow the measured characteristics of the dumbbell's measured expansion and torsion but, alas, yield only alterations of dumbbells lengths smaller than $\pm 3 \mathrm{pm}$ and misorientation angles smaller than $\pm 0.4^{\circ}$ for the image area under investigation [54]. Hence, a more likely explanation could be that the terminating partial dislocation biases the rearrangement of atomic columns in the vicinity of the faulted ribbons and that next but one neighbour interactions between different atomic species will indeed play an important role during this process.

\section{Atomic Structure Of Chromium Implantation Induced LatTice DEFECTS IN GAN (1120)}

As a finale example, we report on dislocations introduced by chromium implantation into GaN. Implantation of transition metals into semiconductors is a candidate process for the fabrication of diluted magnetic semiconductors for spintronic applications [64]. A by-product of the implantation is the creation of ion-induced lattice defects: Extrinsic and intrinsic basal plane stacking faults, bounded by Frank partial or Shockley-Frank partial dislocations, are formed as the result of the precipitation of excess interstitials $[54,65]$.

The GaN films presented in this study were grown by metal organic chemical vapour deposition on (0001) oriented sapphire substrates under Si doping to a concentration of $2 \times 10^{17} \mathrm{~cm}^{-3}$. Cr+ ions were implanted with an energy of $200 \mathrm{kV}$ and a dose of $5 \times 10^{16} \mathrm{~cm}^{-2}$ at a temperature of $350{ }^{\circ} \mathrm{C}$ to avoid amorphization in the asimplanted samples. A high density of basal plane stacking faults is observed besides spherical Cr-rich precipitates in surface near regions after chromium implantation and rapid thermal annealing at $700{ }^{\circ} \mathrm{C}$ for $5 \mathrm{~min}$ in $\mathrm{N}_{2}$ atmosphere [66].

The majority of the basal plane stacking faults is extrinsic bound by Frank partial dislocations with Burgers vector $b=1 / 2$ [0001]. Figure 6 displays the amplitude image $A(r)$ and phase image $\Phi(\mathbf{r})$ of a perfect nanometre sized interstitial loop in the 
end of range depth of implantation. Gallium and nitrogen columns at a dumbbell distance of $114 \mathrm{pm}$, considerably smaller than the information limit of the instrument, are not fully resolved. Nonetheless the N-polarity is unambiguously visible from the directly interpretable bright contrast in the phase image. The tetrahedral coordination across the faulted layer stacking is confirmed, asserting that no foreign chromium-gallium alloy phase is connected with the planar defects.

A magnified view of the core of a Frank partial dislocation at the terminating zone of a several $10 \mathrm{~nm}$ large dislocation loop is shown in the $Z_{\text {opt }}$ image and the $\Phi(\mathbf{r})$ image retrieved from a corresponding focal series displayed in Figure 7(a) and (b). Although the $I(\mathbf{r})$ and $\Phi(\mathbf{r})$ appear very similar at a first glance, the improved signal-to-noise ratio in the phase image enhances the visibility of the nitrogen positions and the dumbbell orientation up to the core of the dislocation. The phase image $\Phi(\mathbf{r})$ fortifies the presence of further nitrogen atoms within the core surrounded by the cage of the five marked gallium columns. The faint phase shift in $\Phi(\mathbf{r})$, in which non-linear image components and image delocalization are eliminated, indicates a nitrogen filled core in favour of a chromium or gallium rich core.

Based on these observations a tentative 5/7 ring configuration for the core is presented in Figure 7(c). The nitrogen atoms shared by the 5 - and 7-membered rings are threefold coordinated with Ga and N. Due to the wrong bond and the presence of unpaired electrons, this structure is not expected to be the most stable configuration. Since $\Phi(\mathbf{r})$ is retrieved from a focal series taken over a period of a few ten seconds, the image conceivably reflects a transient state of the core that gathers further interstitials during observation. The $Z_{\text {opt }}$ image level may therefore contain complementary but not necessarily identical information when compared to $\Phi(\mathbf{r})$. Indeed for the present case the $Z_{\text {opt }}$ image with its inferior noise level compared to $\Phi(\mathbf{r})$ also complies an alternative structure which contains two wrong Ga-Ga bonds connecting threefold coordinated Ga atoms at positions 1-5 and 2-3, respectively.

\section{CONCLUSIONS}


In summary, a combination of negative spherical aberration imaging and the restoration of the exit-plane wavefunction from a focal series of micrographs has been applied for the investigation of lattice imperfections in semiconductor materials at atomic resolution and, in particular, for the extraction of quantitative information on local lattice distortions.

It has been demonstrated that recent improvements in the resolution power of transmission electron microscopes enable the imaging of finest structure details at directly interpretable contrast features also coming along with a widely minimized image delocalization when micrographs are taken under optimized imaging conditions.

Additionally, the numerical retrieval of the exit-plane wavefunction allows for the elimination of artificial contrast features still visible in micrographs taken under optimized focusing conditions. Beyond it's genuine purpose, the retrieval of the exitplane wavefunction was demonstrated to be a most suitable tool for the measurement and numerical correction of residual lens aberrations as well as for proper orientation of specimens during operation of the electron microscope.

Above and beyond the individual vantages coming along with either technique their combined use allows for the accurate identification of lattice imperfections in semiconductor materials at hitherto unequalled imaging. Experimental analyses conspicuously benefit from putting to use aberration corrected input data, i.e. from an increase of the instrumental information limit together with a decrease of image delocalization and a pre-reduction of lens aberrations, as well as from the complete elimination of residual aberrations by the numerical retrieval of the exit-plane wavefunction, which is also free from non-linear imaging artefacts and yields images characterized by a decent signal-to-noise ratio.

By means of the deliberated combination of both techniques, Lomer dislocation cores at $\operatorname{In}_{0.3} \mathrm{Ga}_{0.7} \mathrm{As} / \mathrm{GaAs}$ heterointerfaces have been imaged at atomic resolution and directly identified as being composed of a mirror symmetric ten-atomic ring, thus rendering extensive image simulations superfluous. The quantification of local lattice distortions in the vicinity of a double stacking fault ribbon in GaAs by means of the analysis of experimental phase images $\Phi(\mathbf{r})$ brought clear evidence of an anti- 
symmetric torsion and expansion of atomic dumbbells with respect to the faulted ribbon. A similar quantitative analysis focusing on lattice imperfections in $\mathrm{GaN}$ at atomic resolution based on the synergetic combination of negative spherical aberration corrected imaging and the numerical retrieval of the exit-plane wavefunction is presently merely hampered by the instrumental resolution but will come along with forthcoming aberration corrected sub-Ångström resolution microscopes.

\section{ACKNOWLEDGEMENTS}

The authors are grateful to Arno Förster and Vitaly Guzenko for making available the samples investigated in this study as well as Doris Meertens for indefatigable specimen preparation work. 


\section{FIGURE CAPTIONS}

Figure 1: Partially coherent contrast transfer function (PC CTF) together with the coherent transfer function (CTF) and the damping envelope (DE) assuming parameters $\lambda=2.51 \mathrm{pm}, \mathrm{C}_{\mathrm{S}}=-40.6 \mu \mathrm{m}, Z=11.6 \mathrm{~nm}$ as well as $0.2 \mathrm{mrad}$ for the semi-angle of beam convergence and $6.4 \mathrm{~nm}$ for the half-width of the Gaussian spread of defocus. Arrows indicate positions of crystalline reflections in GaAs.

Figure 2: Analysis of a Lomer type dislocation with $\mathbf{b}=a / 2$ [1ํㅣㅇ at an $\operatorname{In}_{0.3} \mathrm{Ga}_{0.7} \mathrm{As} / \mathrm{GaAs}$ heterointerface. a: Optimum focus micrograph $\mathbf{I}(\mathbf{r})$ taken along the $[1 \overline{1} 0]$ zone axis orientation of the sample. b: "Local" diffraction pattern evaluated from the exit-plane wavefunction of the image area. c: Restored phase image $\Phi(\mathbf{r})$ and d: amplitude image A(r). e: Magnified clipping of the GaAs related image area together with the associated phase images $\Phi_{\text {unc }}(\mathbf{r})$ and $\Phi(\mathbf{r})$ obtained when neglecting and considering measured residual lens aberrations, respectively.

Figure 3: Dislocation core models for symmetrical Lomer dislocations. a: Model retrieved from the exit-plane wavefunction displayed in Figure 2 together with b: the symmetrical glide set type core model according to Hornstra [34].

Figure 4: Analysis of an extrinsic stacking fault in GaAs bound by two adjacent $30^{\circ}$ partial dislocations with $b_{\text {proj }}=[\overline{1} 12]$ in total. a: High-resolution micrograph $I(r)$ taken under $Z_{\text {opt }}$ conditions. b: Phase image $\Phi(\mathbf{r})$ and c: amplitude image $A(r)$ calculated from the associated focal series of micrographs. d: Magnified clipping of $\Phi(\mathbf{r})$ with the positions of atomic columns superimposed and the dislocation core positions of $30^{\circ}$ partials indicated by red circles. e: Contour representation of the lattice displacements along the [1 $1 \overline{1} 2]$ direction with reference to the dashed red framed area. f: Displacement profile measured perpendicular to the faulted ribbon at the area indicated by the dashed blue frame.

Figure 5: Locally inhomogeneous distortions of the atomic dumbbells in the vicinity of the faulted double ribbon. a: Clipping from the phase image displayed in Figure 4(b) with atomic column positions superimposed and dumbbell distortions indicated exemplarily in dependence on specific positions along the [111] direction. 
b: Average projected bond length $\langle d\rangle$ and $\mathbf{c}$ : misorientation angle $\langle\delta$ of the dumbbells along the (111) direction. The lattice planes belonging to the double stacking fault ribbon are indicated in lighter grey colour.

Figure 6: Interstitial loop in Cr-implanted GaN viewed along the [11 $\overline{2} 0]$ direction. a: Amplitude image $\mathrm{A}(\mathbf{r})$ and b: phase image $\Phi(\mathbf{r})$ calculated from a focal series of micrographs. Simulated images of the periodic N-polarity GaN structure assuming a sample thickness of $3.2 \mathrm{~nm}$ at a resolution of $7.5 \mathrm{~nm}^{-1}$ are superimposed in the lower left part of the images. The stacking sequence is $A B$ ABCABAB, where the underlined part highlights sphalerite stacking.

Figure 7: Frank partial dislocation with the Burgers vector $\mathbf{b}=1 / 2$ [0001] projected along the [11 $\overline{2} 0$ ] direction. a: Experimental micrograph $I(r)$ taken under $Z_{\text {opt }}$ conditions and b: correspondingly retrieved phase image $\Phi(\mathbf{r})$. c: 5/7 ring configuration for the core of the Frank partial dislocation. 


\section{REFERENCES}

[1] H. Alexander and H. Teichler in Handbook of Semiconductor Technology, Vol 1, edited by K. A. Jackson and W. Schröter (Wiley-VCH, New York (U.S.), 2000), pp. 291-376.

[2] R. Jones, Mater. Sci. Eng. B71 24 (2000).

[3] P. B. Hirsch, Mater. Sci. Technol., 1(9) 666 (1985).

[4] S. P. Beckmann and D. C. Chrzan, Physica B 340-342 1001 (2003).

[5] C. Kisielowski, C. J. D. Hetherington, Y. C. Wang, R. Kilaas, M. A. O'Keefe, and A. Thust, Ultramicroscopy 89243 (2001).

[6] M. A. O'Keefe, E.C. Nelson, E.C. Wang, and A. Thust, Phil. Mag. B 711861 (2001).

[7] B. Freitag, S. Kujawa, P. M. Mul, J. Ringnalda, and P. C. Tiemeijer, Ultramicroscopy 102209 (2005).

[8] C. L. Jia, M. Lentzen, and K. Urban, Science 299870 (2003).

[9] C. L. Jia, M. Lentzen, and K. Urban, Microscopy and Microanalysis 10174 (2004).

[10] J. L. Hutchison, J. M. Titchmarsh, D. J. H. Cockayne, R. C. Doole, C. J. D. Hetherington, A. I. Kirkland, and H. Sawada, Ultramicroscopy 1037 (2005).

[11] H. Lichte, Ultramicroscopy 3813 (1991).

[12] W. Coene and A. J. E. M. Jansen, Scan. Microsc. Suppl. 6379 (1992).

[13] H. Rose, Optik 8519 (1990).

[14] M. Haider, H. Rose, S. Uhlemann, E. Schwan, B. Kabius, and K. Urban, Nature 392768 (1998).

[15] M. Lentzen, B. Jahnen, C. L. Jia, A. Thust, K. Tillmann, and K. Urban, Ultramicroscopy 92233 (2002).

[16] W. M. J. Coene, G. Janssen, M. Op de Beeck, and D. van Dyck, Phys. Rev. Lett. 693743 (1992). 
[17] W. M. J. Coene, A. Thust, M. Op de Beeck, and D. van Dyck, Ultramicroscopy 64109 (1996).

[18] A. Thust, W. M. J. Coene, M. Op de Beeck, and D. van Dyck, Ultramicroscopy 64211 (1996).

[19] A. I. Kirkland, W. O. Saxton, K. L. Chau, K. Tsuno, and M. Kawasaki, Ultramicroscopy 57355 (1995).

[20] A. I. Kirkland, W. O. Saxton, and G. Chand, J. Electron Microsc. 111 (1997).

[21] A. Thust, M. H. F. Overwijk, W. M. J. Coene, and M. Lentzen, Ultramicroscopy 64249 (1996).

[22] A. Thust, C. L. Jia, and K. Urban, in Proceedings ICEM-15, Vol. 1, edited by R. Cross (Microscopy Society of Southern Africa, Durban, 2002), pp. 167-168.

[23] R. R. Meyer, A. I. Kirkland, and W. O. Saxton, Ultramicroscopy 9289 (2002).

[24] R. R. Meyer, A. I. Kirkland, and W. O. Saxton, Ultramicroscopy 99115 (2004).

[25] D. B. Williams and C. B. Carter, Transmission Electron Microscopy (Plenum Press, New York and London, 1996).

[26] M. Lentzen, Ultramicroscopy 99211 (2004).

[27] S. Uhlemann and M. Haider, Ultramicroscopy 72109 (1998).

[28] M. A. O'Keefe, C. J. D. Hetherington, Y. C. Wang, E. C. Nelson, J. H. Turner, C. Kisielowski, J. O. Malm, R. Mueller, J. Ringnalda, M. Pan, and A. Thust, Ultramicroscopy 89215 (2001).

[29] L. Y. Chang, F. R. Chen, A. I. Kirkland, and J. J. Kai, J. Electr. Microsc. 52 359 (2003).

[30] R. R. Meyer, A. I. Kirkland, and W. O. Saxton, Ultramicroscopy 99115 (2004).

[31] K. Tillmann, A. Thust, and K. Urban, Microscopy and Microanalysis 10185 (2004).

[32] F. Zemlin, K. Weiss, P. Schiske, W. Kunath, and K. H. Herrmann, Ultramicroscopy 349 (1978).

[33] W. M. Lomer, Phil. Mag. 421327 (1951). 
[34] J. Hornstra, J. Phys. Chem. Solids 5129 (1958)

[35] A. Bourret, J. Dessaux and R. Renault, Phil. Mag. A 451 (1982).

[36] A. Vilà, A. Cornet, J. R. Morante, P. Ruterna, M. Loubradou, R. Bonnet, Y. González, and L. González, Phil. Mag. A 7585 (1995).

[37] D. Wang, H. Chen, F. H. Li, K. Kawasaki, T. Oikawa, Ultramicroscopy 93139 (2002).

[38] J. N. Stirman, P. A. Crozier, D. J. Smith, F. Phillipp, G. Brill and S. Sivananthan, Appl. Phys. Lett. 842530 (2004).

[39] K. Tillmann and A. Förster, Thin Solid Films 36893 (2000).

[40] L. Houben, A. Thust, and K. Urban, Ultramicroscopy 106200 (2006).

[41] S. Lopatin, S. J. Pennycook, J. Narayan, and G. Duscher, Appl. Phys. Lett. 81 2728 (2002).

[42] F. C. Frank, Phil. Mag. 42809 (1951).

[43] I. L. F. Ray and D. J. H. Cockayne, Proc. Roy. Soc. A 325543 (1971).

[44] P. Gai and A. Howie, Phil. Mag. 30939 (1974).

[45] S. Amelinckx, in Dislocations in Solids, Vol. 2, edited by F.R.N. Nabarro (NorthHolland, Amsterdam, 1979), pp. 67-460.

[46] A. Olsen and J. C. H. Spence, Phil. Mag. A 43945 (1981).

[47] M. Tanaka and B. Jouffrey, Phil. Mag. A 50733 (1984).

[48] B. C. de Cooman and D.B. Carter, phys. stat. sol. (a) 112473 (1989).

[49] R. W. Nunes, J. Bennetto, and D. Vanderbilt, Phys. Rev. B 5812563 (1998).

[50] J. F. Justo, R. W. Nunes, and L. V. C. Assali, J. Phys.: Condens. Matter 14 12749 (2002).

[51] S. P. Beckman, X. Xu, P. Specht, E. R. Weber, C. Kisielowski, and D. C. Chrzan, J. Phys.: Condens. Matter 1412673 (2002).

[52] H. R. Kolar, J. C. H. Spence, and H. Alexander, Phys. Rev. Lett. 774031 (1996). 
[53] X. Xu, S. P. Beckmann, P. Specht, E. R. Weber, D. C. Chrzan, R. P. Ernie, I. Arslan, N. Browning, A. Bleloch, and C. Kisielowski, Phys. Rev. Lett. 95 145501 (2005).

[54] J. P. Hirth and J. Lothe, Theory of Dislocations, (McGraw Hill, New York (U.S), 1968).

[55] M. J. Hÿtch, E. Snoeck, and R. Kilaas, Ultramicroscopy 74131 (1998).

[56] J. L. Rouvière and E. Sarigiannidou, Ultramicroscopy 1061 (2005).

[57] C. L. Jia and A. Thust, Phys. Rev. Lett. 825052 (1999).

[58] M. J. Hÿtch, J. L. Putax, and J. M. Penisson, Nature 423279 (2003).

[59] A. M. Gomez and P. B. Hirsch, Phil. Mag. A 38733 (1978).

[60] K. Tillmann, M. Lentzen, and R. Rosenfeld, Ultramicroscopy 83111 (2000).

[61] R. Kilaas, S. Paciornik, A. J. Schwartz, and L. E. Tanner, Journal of ComputerAssisted Microscopy 6129 (1994).

[62] H. Seitz, K. Ahlborn, M. Seibt, and W. Schröter, J. Microsc. 190184 (1997).

[63] S. H. Lim, D. Shindo, I. Yonenaga, P. D. Brown, and C. J. Humphreys, Phys. Rev. Lett. 815350 (1998).

[64] H. Ohno, Science 281951 (1998).

[65] V. Kirchner, H. Heinke, U. Birkle, S. Einfeld, D. Selke, and P. L. Ryder, Phys. Rev. B 5815749 (1998).

[66] V. A. Guzenko, N. Thillosen, A. Dahmen, R. Calarco, Th. Schäpers, L. Houben, M. Luysberg, B. Schineller, M. Heuken, and A. Kaluza, J. Appl. Phys. 96 5663 (2004). 